\title{
Channel Estimation and Peak-to-Average Power Ratio Analysis of Narrowband Internet of Things Uplink Systems
}

\author{
Md Sadek Ali (D), Yu Li, Md Khalid Hossain Jewel, \\ Oluwole John Famoriji $\mathbb{D}$, and Fujiang Lin
}

Micro-/Nano-Electronic System Integration R\&D Center (MESIC), Department of Electronic Science and Technology, University of Science and Technology of China (USTC), Hefei, Anhui, 230026, China

Correspondence should be addressed to Md Sadek Ali; sadek@mail.ustc.edu.cn

Received 27 November 2017; Accepted 21 May 2018; Published 5 July 2018

Academic Editor: Pavlos I. Lazaridis

Copyright (c) 2018 Md Sadek Ali et al. This is an open access article distributed under the Creative Commons Attribution License, which permits unrestricted use, distribution, and reproduction in any medium, provided the original work is properly cited.

\begin{abstract}
Narrowband Internet of Things (NB-IoT) is a cellular based promising low-power wide-area network (LPWN) technology standardized by the 3rd Generation Partnership Project (3GPP) in release-13 as a part of the future 5th Generation (5G) wireless communication systems. The main design target of NB-IoT was to enhance radio coverage by repeating signal over an additional period of time for the ultralow-end IoT devices that would be operated in extreme coverage environments. But the power efficiency of the low-cost NB-IoT user equipment (NB-IoT UE) in the uplink is the major concern. Coverage improvement from signal repetitions depends on the channel estimation quality at extremely bad radio conditions. The typical operating signal-to-noise ratio (SNR) for NB-IoT is expected to be much lower than the zero. In this paper, we have proposed two efficient narrowband demodulation reference signal (NDMRS)-assisted channel estimation algorithms based on the conventional least squares (LS) and minimum mean square error (MMSE) estimation methods. The theoretical analysis and the link-level performance of our proposed estimation methods are presented. Simulation results exhibit that the proposed methods provide better estimation precision compared to the traditional LS and MMSE methods at the low SNR situations. Furthermore, we have analyzed the raisedcosine (RC) and square-root-raised cosine (RRC) pulse shaping to reduce peak-to-average power ratio (PAPR) as an uplink transmit filter. The PAPR values are evaluated through extensive computer simulations for both single-tone and multi-tone transmissions. Our evaluation results vindicate that the RRC pulse shaping with lower PAPR values is feasible to design of practical NB-IoT uplink transmitter and increases power efficiency.
\end{abstract}

\section{Introduction}

The Internet of Things (IoT) is a novel prototype which offers massive connectivity to physical objects, radio-frequency identification (RFID) tags, vehicles, sensors, actuators, and other things embedded with electronics to the Internet. Io $\mathrm{T}$ allows things to be connected across existing network infrastructure, interacting with each other through unique addressing schemes, thus reducing extra deployment cost and improving accuracy and efficiency. According to [1], there will be more than 30 billion devices connected wirelessly to the IoT by 2020. Nokia [2], based on Machina research 2015 , predicted that about 30 billion connected IoT devices will be deployed by 2025 , of which cellular IoT (CIoT) and low-power wide-area (LPWA) modules are about 23 percent. Traditionally, mobile broadband networks need high throughput and low latency, whereas LPWA applications require low-throughput, extended coverage, low-cost, low complexity, scalability, low delay sensitivity, and high power efficiency [3-6].

There are many short-range wireless communication technologies $[7,8]$ like Bluetooth low energy (BLE), Wi-Fi, $\mathrm{Li}-\mathrm{Fi}$, ZigBee, and Z-wave, to enable the IoT. Some of the IoT enabling technologies $[9,10]$ such as SigFox and LoRa that are operating in license-exempted band (i.e., industrial, scientific, and medical (ISM) band). On the other hand, Global System for Mobile Communications (GSM) and the 3rd Generation Partnership Project (3GPP) standard LongTerm Evolution (LTE) are operating in licensed spectrum to enable the IoT. A new cellular based IoT enabling technology 
named narrowband IoT (NB-IoT) was specifically designed for ultralow-end IoT applications. The 3GPP finalized the specifications of NB-IoT in LTE release-13 [11]. It is secure and reliable for data transmission because of the deployment in licensed spectrum of GSM or LTE [12]. NB-IoT enables IoT whose applications are very diverse including smart metering, smart cities, smart water, smart environment, smart agriculture, smart animal firming, retail, logistics, security and emergencies, industrial control, and domestic and home automation. Therefore, the channel condition of NB-IoT is very complicated due to its diverse application scenarios.

Since NB-IoT is still in its infancy, the proper guidelines for efficient channel estimation and equalization are still missing in the current literature. Channel estimation based on the pilot signal has been studied well for traditional orthogonal frequency division multiplexing (OFDM) and single-carrier frequency division multiple access (SCFDMA) systems, e.g., $[13,14]$. In the literature, most of the NB-IoT research focused on frame structure design [15], scheduling and link adaptation [16], random access procedure [17], and system acquisition [18]. Positioning performance of NB-IoT has been studied in $[19,20]$. Y. D. Beyene et al. in [21] have investigated the performance of three traditional channel estimators only for $15 \mathrm{kHz}$ subcarrier spacing. L. Zhang et al. in [22] have analyzed the channel equalization and coexistence problem of NB-IoT and LTE signals using only traditional LTE supported subcarrier spacing. To the best of our knowledge, the channel estimation of NB-IoT uplink with $3.75 \mathrm{kHz}$ subcarrier spacing has not been studied yet. Thus, efficient channel estimation is the prerequisite of coverage improvement, equalization, and signal decoding at the receiver.

In NB-IoT uplink, low peak-to-average power ratio (PAPR) enhances the efficiency of the low-cost power amplifier. Low out-of-band radiation is desired for the uplink transmitter due to its very narrow bandwidth. The PAPR problem is more of a concern in the NB-IoT uplink (i.e., low-cost transmitter). The efficiency of the power amplifier is crucial for the low-cost NB-IoT user equipment (NB-IoT UE) with limited battery power. Thus, low PAPR in the NB-IoT uplink is ultimate desire owing to low-cost power amplifier. PAPR reduction techniques such as scrambling, discrete Fourier transform (DFT) spreading, and cyclic prefix (CP) insertion can be applied at the uplink transmitter. NB-IoT supports modulation schemes like $\pi / 2$-BPSK (binary phase shift keying) and $\pi / 4$-QPSK (quadrature phase shift keying), which are also robust against PAPR by applying constellation rotation to make smooth transition between constellation points. However, these techniques are not fully preserved. Further reduction of PAPR would be great demand at the uplink because transmitter is the low-cost and low-power IoT device. In [23], the authors have been evaluated the PAPR values employing root-raised cosine (RRC) pulse shaping (PS) filter only for the single-tone transmission. This is the first time that the partial analysis of PAPR was taken into account in the NB-IoT uplink transmitter.

In this paper, we have developed an uplink NB-IoT system model according to the $3 \mathrm{GPP}$ specifications in release13 [24-26]. The motivation was that the uplink transmission of NB-IoT systems is more complicated compared to the downlink transmission. We have considered both types of transmission schemes and subcarrier spacing for channel estimation and PAPR analysis. The major contributions of this paper can be summarized as follows:

(1) We have provided a brief overview of NB-IoT technology including deployment options, physical channels and signals, uplink frame structure, and resource unit (RU) definition. An analytic NB-IoT uplink received signal model is derived as a function of transmitted signal and channel impairments. Narrowband demodulation reference signal (NDMRS) generation and mapping to time-frequency grid is also presented.

(2) We have proposed two NDMRS-aided channel estimation algorithms based on the traditional least squares (LS) and minimum mean square error (MMSE) estimators that can be coped with the complicated channel conditions of NB-IoT systems. Through simulations, we have investigated and verified the effectiveness of our proposed algorithms compared with the conventional LS and MMSE algorithms in terms of bit error rate (BER) related to signal-to-noise ratio (SNR). Simulation results show that our proposed channel estimation algorithms outperform the others.

(3) In addition, we have provided theoretical analysis of PAPR for the NB-IoT uplink employing raised-cosine (RC) and square-root-raised cosine (RRC) pulse shaping filters. We have also showed the comparison of PAPR values that are obtained with and without PS through computer simulations for both singletone and multi-tone transmissions. Numerical results elucidate that RRC PAPR reduction technique is feasible for the implementation of NB-IoT uplink transmitter.

The rest of the paper is organized as follows: in Section 2, a brief overview of the NB-IoT technology is provided. NB-IoT uplink signal model and NDMRS generation and mapping are presented in Section 3. Theoretical analysis of channel estimation and its performance analysis by simulations are given in Section 4. In Section 5, PAPR reduction techniques and its numerical results are presented. Finally, we conclude the paper in Section 6.

Notations. Bold face lowercase letters are used to represent time-domain vectors (or matrices), while frequencydomain vectors/matrices are denoted by uppercase bold characters. Superscripts $(\cdot)^{T}$ and $(\cdot)^{H}$ denote the transpose and Hermitian of a vector, a scalar, or a matrix, respectively, and $(\cdot)^{-1}$ denotes matrix inversion. The circular convolution operation is denoted by $\otimes$ and the operators $\mathrm{E}[\cdot],|\cdot|$, and $\|\cdot\|$ represent the expectation, absolute value, and Euclidean norm, respectively. $\boldsymbol{I}_{L}$ denotes the $L \times L$ identity matrix.

\section{Overview of NB-IoT Technology}

NB-IoT was designed by $3 \mathrm{GPP}$ as a key technology to meet the demands of massive low-power IoT connectivity for the 


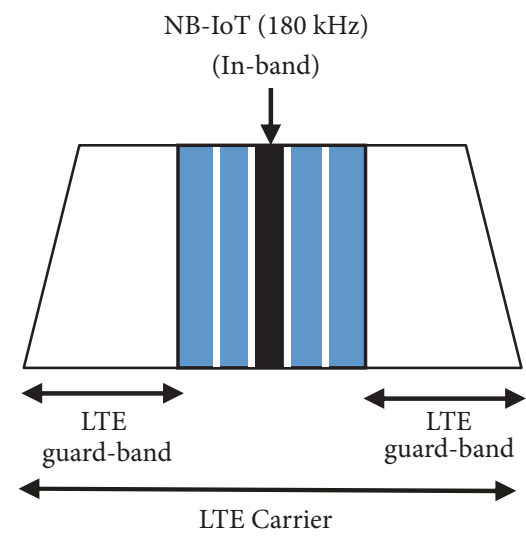

(a)

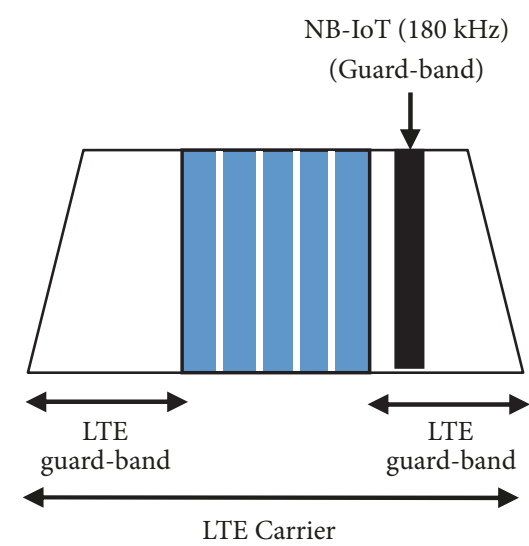

(b)

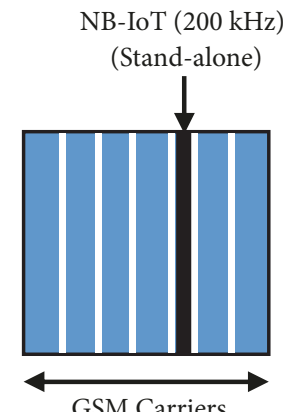

GSM Carriers

Figure 1: NB-IoT modes of operation: (a) in-band, (b) guard-band, and (c) standalone.

evolution of future $5 \mathrm{G}$ wireless communication systems. It can be implemented in three different operation modes specified by 3GPP in release-13 [27, 28]: standalone, in-band, and guard-band. The illustrations of three deployment options are given in Figure 1. NB-IoT can be deployed by replacing one or more efficiently reframed $200 \mathrm{kHz}$ GSM carriers, a so-called standalone mode of operation. Radio coverage for NB-IoT can be enhanced significantly by using all the transmit power at the evolved node B (eNB) also known as base station. In in-band operation, it can be implemented inside the LTE carrier using one or more physical resource blocks (PRBs); a PRB corresponds to $180 \mathrm{kHz}$ bandwidth. LTE and NB-IoT share the total transmit power at the eNB. Wide-area coverage can also be achieved by boosting power on the NB-IoT PRB. The spectrum efficiency can also be increased by the sharing of PRB between LTE and NB-IoT. The third option can be deployed within the LTE carrier's unused guard-band. This is allowed only for $5 \mathrm{MHz}$ or higher LTE system bandwidth. In-band and guard-band deployments of NB-IoT reuse the existing LTE base station's radio-frequency (RF) front-end and the baseband numerologies with some modifications to fit into the narrow bandwidth [29]. The coexistence of LTE and NB-IoT has been investigated through rigorous simulations in [27]. Thus, it will not incur extra deployment cost and time to come in operation. The modes of operation should be known to the NB-IoT UE when it is turned on and searches for an NB-IoT carrier. NB-IoT supports $100 \mathrm{kHz}$ channel raster for all types of operation modes.

NB-IoT channels and signals are designed based on the existing LTE channels and signals with required modifications and simplifications to fit the 3GPP specified 180 $\mathrm{kHz}$ narrow bandwidth. The NB-IoT downlink and uplink channels and signals with their functions according to release-13 are given in Table 1 . The 3GPP is specified as narrow as system bandwidth of $180 \mathrm{kHz}$ for both downlink and uplink transmissions. NB-IoT supports only frequency division duplexing (FDD) with half duplex transmission. In the downlink, NB-IoT inherits the downlink numerology from existing LTE although with further restricted support. It uses OFDM with $15 \mathrm{kHz}$ subcarrier spacing as in LTE. The basic time unit for NB-IoT is specified by a factor of $T_{\mathrm{s}}=$ $1 /(15000 \times 2048)$ seconds. The slot duration $T_{\text {slot }}=15360 \times$ $T_{\mathrm{s}}=0.5 \mathrm{~ms}$. A pair of consecutive slots constitute a subframe with duration $1 \mathrm{~ms}$. The NB-IoT radio frame for downlink consists of 10 subframes with duration $T_{\mathrm{f}}=307200 \times T_{\mathrm{s}}=10$ ms. Thus, a radio frame contains 20 slots; the slot number within a radio frame is denoted here as $n_{\mathrm{s}}$, where $n_{\mathrm{s}} \in$ $\{0,1, \cdots, 19\}$. In NB-IoT uplink, two schemes are supported for the baseband modulation: single-tone transmission based on frequency division multiple access (FDMA) and multitone (i.e., 3, 6, or 12 subcarriers) transmission according to SC-FDMA. Two different subcarrier spacing types are allowed for single-tone transmission: $15 \mathrm{kHz}$ and $3.75 \mathrm{kHz}$ [25]. NB-IoT with $3.75 \mathrm{kHz}$ subcarrier spacing is designed to provide more capacity in power limited scenarios [27]. The first one is the same as in LTE with $0.5 \mathrm{~ms}$ slot, 1 ms subframe, and $10 \mathrm{~ms}$ frame in time-domain, whereas there are 12 subcarriers within $180 \mathrm{kHz}$ system bandwidth in frequency-domain. A time-frequency grid structure for $15 \mathrm{kHz}$ subcarrier spacing within a frame is illustrated in Figure 2. On the other hand, the second one is different from LTE with slot duration $T_{\text {slot }}=61440 \times T_{\mathrm{s}}=2 \mathrm{~ms}$. Thus, five consecutive slots constitute a radio frame with the duration of $61440 \times T_{\mathrm{s}} \times 5=10 \mathrm{~ms}$, where the slot number $n_{\mathrm{s}}$ within a radio frame can be selected from the set $n_{\mathrm{s}} \in\{0,1, \cdots, 4\}$. In the frequency-domain, $180 \mathrm{kHz}$ system bandwidth consists of 48 subcarriers. Figure 3 shows the NB-IoT uplink resource grid structure within a frame for $3.75 \mathrm{kHz}$ subcarrier spacing. For single-tone transmission both subcarrier spacing types can be used, whereas only $15 \mathrm{kHz}$ subcarrier spacing is specified for multi-tone transmission.

The 3GPP in [24] defined a new feature for NB-IoT uplink called resource unit (RU), which is the basic unit for narrowband physical uplink shared channel (NPUSCH) allocation. In the time-domain, one transport block size (TBS) can be mapped to multiple RUs from the set $\{1,2,3,4,5,6,8,10\}$. In 3GPP [25], TBSs are defined as a function of the number of RUs and modulation and coding scheme (MCS) level. The maximum TBS is 1000 bits for the uplink. The characterization of a RU is given in Table 2. In NB-IoT 
TABLE 1: Physical channels and signals for NB-IoT systems.

\begin{tabular}{|c|c|c|}
\hline & Channels/Signals & Functions \\
\hline \multirow{4}{*}{ Downlink } & $\begin{array}{l}\text { Narrowband Physical Downlink Control Channel } \\
\text { (NPDCCH) }\end{array}$ & $\begin{array}{l}\text { Scheduling information for both downlink and uplink } \\
\text { data channels }\end{array}$ \\
\hline & $\begin{array}{l}\text { Narrowband Physical Downlink Shared Channel } \\
\text { (NPDSCH) }\end{array}$ & Downlink dedicated and common data \\
\hline & Narrowband Physical Broadcast Channel (NPBCH) & Master information for system access \\
\hline & Narrowband Synchronization Signal (NPSS/NSSS) & $\begin{array}{l}\text { Cell search including time and frequency } \\
\text { synchronization and cell identity detection }\end{array}$ \\
\hline \multirow{3}{*}{ Uplink } & $\begin{array}{l}\text { Narrowband Physical Uplink Shared Channel } \\
\text { (NPUSCH) }\end{array}$ & Uplink dedicated data and control information \\
\hline & $\begin{array}{c}\text { Narrowband Physical Random Access } \\
\text { Channel(NPRACH) }\end{array}$ & Random access procedure \\
\hline & Narrowband Demodulation Reference Signal(NDMRS) & Uplink channel estimation \\
\hline
\end{tabular}

TABLE 2: Characterization of resource unit (RU).

\begin{tabular}{lcccc}
\hline Subcarrier spacing & No. of subcarriers & No. of slots & No. of SC-FDMA symbols & Tx time interval (TTI) \\
\hline $3.75 \mathrm{kHz}$ & 1 & 16 & 112 & $32 \mathrm{~ms}$ \\
\hline \multirow{3}{*}{$15 \mathrm{kHz}$} & 1 & 16 & 112 & $8 \mathrm{~ms}$ \\
& 3 & 8 & 56 & $4 \mathrm{~ms}$ \\
& 6 & 4 & 28 & 14 \\
\hline
\end{tabular}

systems, repetition of user data and associated control signal transmission has been taken in the 3GPP as a key technique to achieve wide-area coverage. Repetitions of same signal can be increased transmission reliability but reduced spectral efficiency. Maximum 2048 and 128 repetitions are allowed for downlink and uplink transmissions, respectively, suggesting that the received data would be decoded even when the noise power is far greater than the signal power. In other words, the eNB and NB-IoT UE transmits the same TBS repeatedly as many times as indicated in the downlink and uplink, respectively. The NB-IoT UE and eNB at the receiver combines the repetitions before decoding the transmitted data. The number of repetitions is determined by the eNB and NB-IoT UE to achieve the desired SNR at the NB-IoT UE and eNB receiver, respectively.

\section{NB-IoT Uplink System Model}

The system model of uplink NB-IoT systems with NDMRS sequence, channels, and the associated estimation and equalization blocks is shown in Figure 4. The uplink transmitter comprises transport channel also known as uplink shared channel (UL-SCH) and data channel (NPUSCH) processing. Binary input data arrives to the channel coding unit in the form of one transport block over a number of RUs per uplink cell. The number of RUs is scheduled according to [25]. In 3GPP [30], UL-SCH processing consists of transport block cyclic redundancy check (CRC) attachment (e.g., 24 bits with generator polynomial $\left.\mathrm{g}_{\mathrm{CRC} 24 \mathrm{~A}}(\mathrm{D})\right), 1 / 3$-rate based turbo coding, and rate matching to yield a codeword input to the NPUSCH.
3.1. NDMRS Sequence Generation and Mapping. A NDMRS sequence $\bar{r}_{u}(n)$ can be generated for the case when the number of subcarriers $N_{\mathrm{sc}}^{\mathrm{RU}}=1$ in a RU is as

$$
\begin{aligned}
\bar{r}_{u}(n)=\frac{1}{\sqrt{2}}(1+j)(1-2 c(n)) w & (n \bmod 16), \\
0 & \leq n<M N_{\text {slots }}^{\mathrm{RU}} N_{\mathrm{RU}}
\end{aligned}
$$

where $c(n)$ is the binary sequence defined by a length-31 Gold sequence, $M$ denotes the repetition number of same signal transmissions, $N_{\text {slots }}^{\mathrm{RU}}$ represents number of slots in a RU, and $N_{\mathrm{RU}}$ is the number of RUs. The initialization value of the first sequence is specified with a unit impulse function of length-31. The second sequence is initialized with the seed $c_{\text {init }}=35$ at the start of the NPUSCH transmission [24]. The variable $w(n)$ is defined in [24], where the base sequence index is $u=N_{\mathrm{ID}}^{\mathrm{Ncell}} \bmod 16$ for NPUSCH format-1 without enabling group hopping. Thus, the NDMRS sequence $r_{u}(n)$ for NPUSCH format-1 can then be represented as

$$
r_{u}(n)=\bar{r}_{u}(n)
$$

The NDMRS sequence $r_{u}(n)$ for the number of subcarriers greater than one in a RU is defined by a cyclic shift $\alpha$ of a base sequence as

$$
r_{u}(n)=e^{j \alpha n} e^{j \varphi(n) \pi / 4}, \quad 0 \leq n<N_{\mathrm{sc}}^{\mathrm{RU}}
$$

where $\varphi(n)$ is defined in [24] for the scheduled number of subcarriers in a RU. Without loss of generality, we assume 


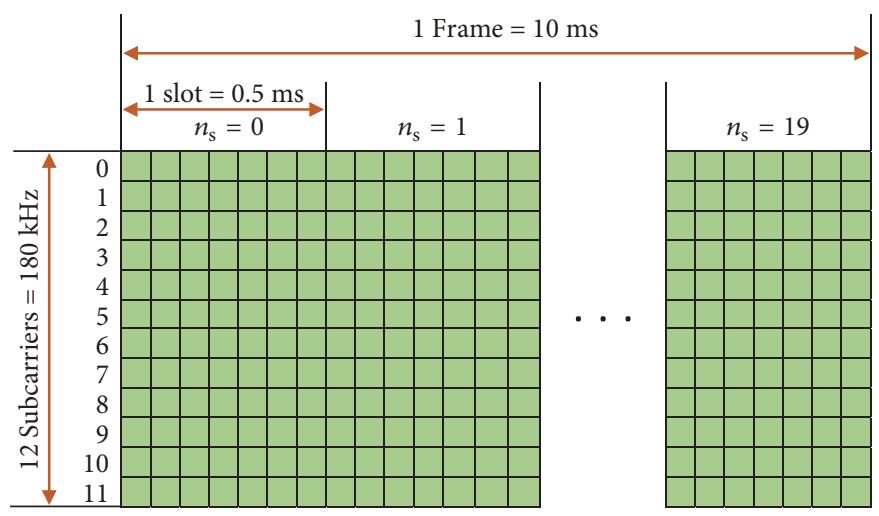

FIgURE 2: NB-IoT uplink resource grid structure for $15 \mathrm{kHz}$ sub-channel bandwidth.

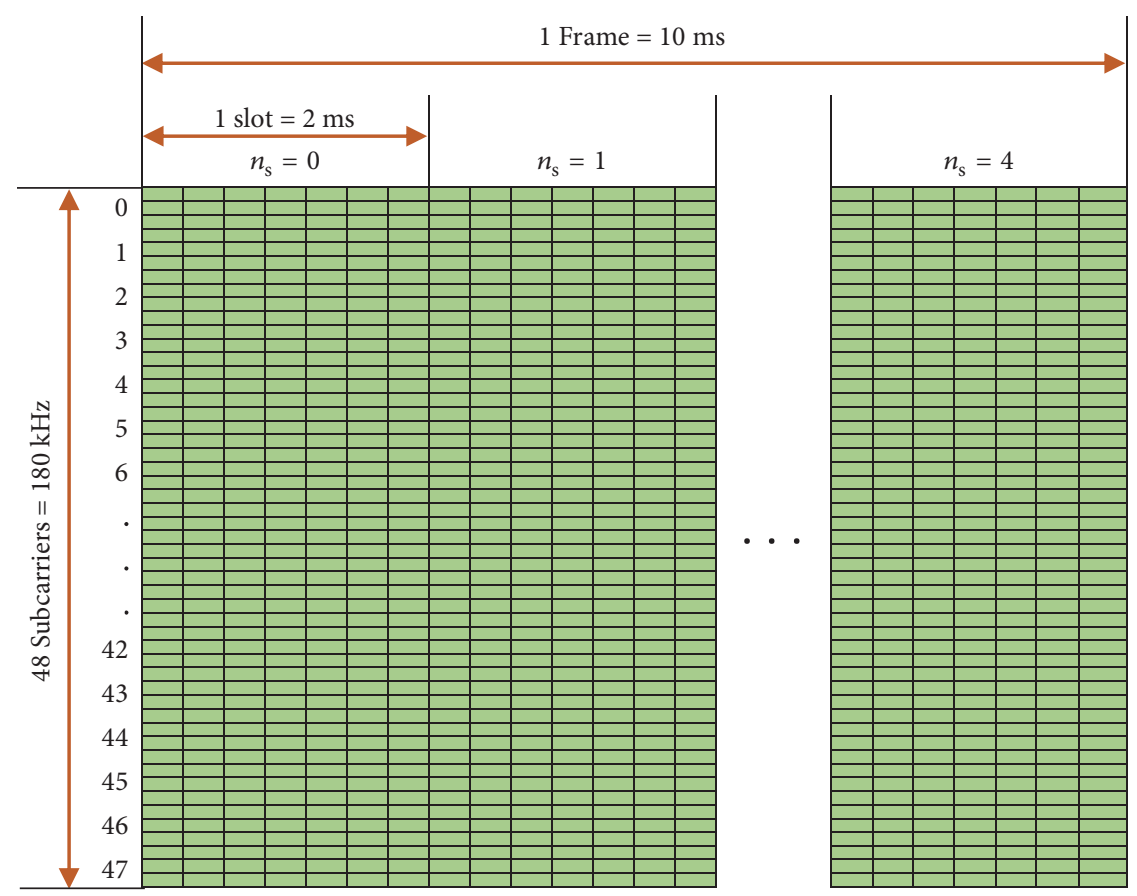

FIgURE 3: NB-IoT uplink resource grid structure for $3.75 \mathrm{kHz}$ sub-channel bandwidth.

that there is no higher layer signaling; then the base sequence index $u$ can be obtained as

$$
u= \begin{cases}N_{\mathrm{ID}}^{\mathrm{Ncell}} \bmod 12 & \text { for } N_{\mathrm{sc}}^{\mathrm{RU}}=3 \\ N_{\mathrm{ID}}^{\mathrm{Ncell}} \bmod 14 & \text { for } N_{\mathrm{sc}}^{\mathrm{RU}}=6 \\ N_{\mathrm{ID}}^{\mathrm{Ncell}} \bmod 30 & \text { for } N_{\mathrm{sc}}^{\mathrm{RU}}=12 .\end{cases}
$$

The cyclic shift $\alpha$ for $N_{\mathrm{sc}}^{\mathrm{RU}}=3$ and 6 is defined in [24], whereas $\alpha=0$ for $N_{\mathrm{sc}}^{\mathrm{RU}}=12$.

The NDMRS sequence is also known as pilot symbol, which is transmitted together with the user data symbols to estimate channel response in uplink NB-IoT systems. In each NB-IoT uplink slot, NDMRS symbols are mapped to the allocated number of subcarriers in a RU of the fourth SCFDMA symbol for $15 \mathrm{kHz}$ subcarrier spacing, whereas the fifth symbol is for $3.75 \mathrm{kHz}$ subcarrier spacing. An inverse discrete Fourier transform (IDFT) operation is performed on the contents of resource grid that contains the NDMRS symbols to convert time-domain reference sequence followed by $\mathrm{CP}$ addition.

3.2. Analytical Uplink Signal Model. Let us consider that an NB-IoT UE transmits a block of bits $\boldsymbol{b}=[b(0), b(1), \ldots$, $\left.b\left(N_{\text {bit }}-1\right)\right]$, where $N_{\text {bit }}$ is the number of transmitted bits in a codeword on the NPUSCH in one subframe. The codeword bits $\boldsymbol{b}$ are scrambled using NB-IoT UE specific scrambling sequence in neighboring cells to ensure that the interference is randomized and the transmission from different cells is separated prior to decoding at the eNB receiver. Thus, we obtain a block of scrambled bits $\widetilde{b}(i)$ as

$$
\tilde{b}(i)=(b(i)+c(i)) \bmod 2
$$




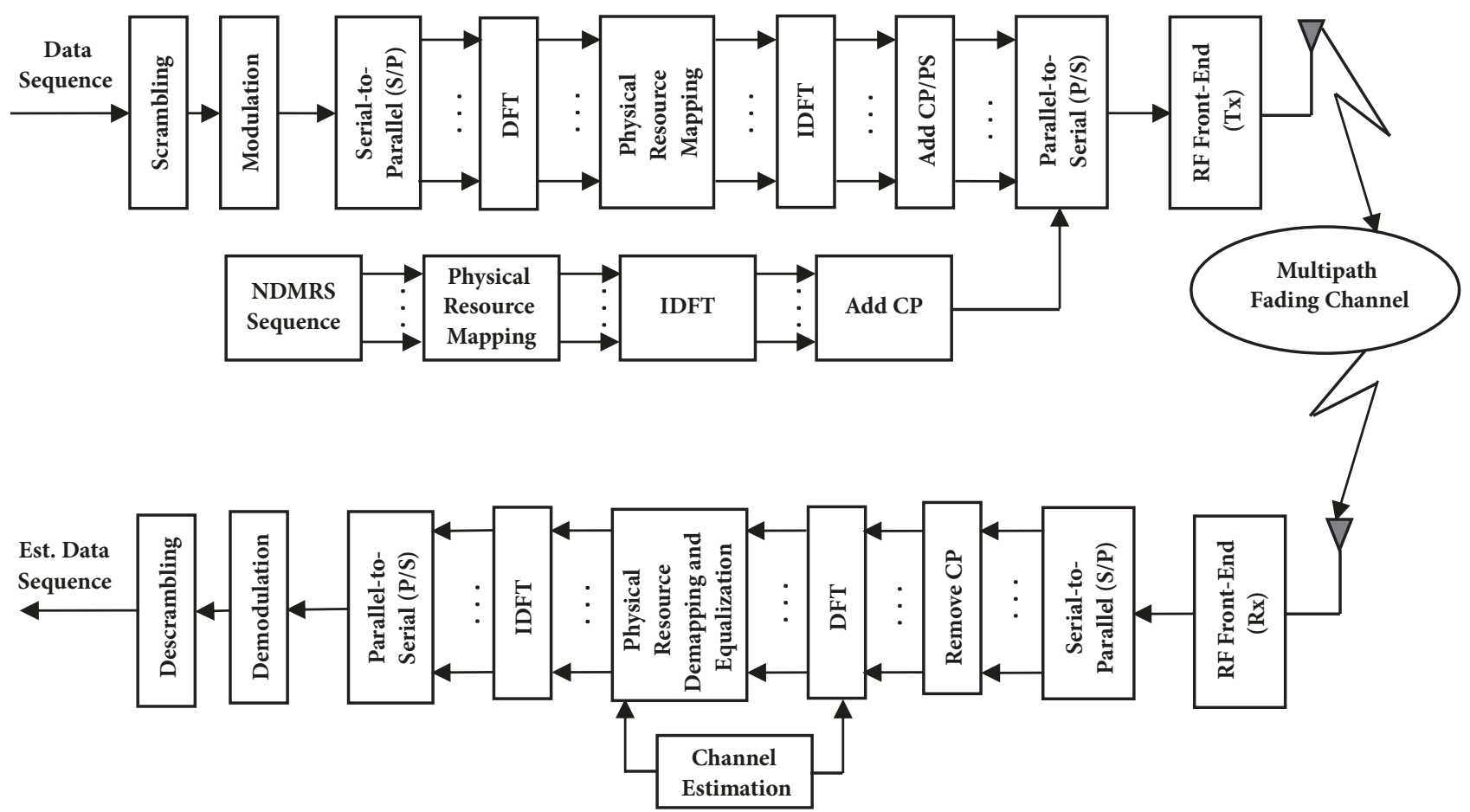

CP: Cyclic Prefix

PS: Pulse Shaping

FIGURE 4: Block diagram of uplink NB-IoT systems.

where $i=0,1, \ldots, N_{\mathrm{bit}}-1$ and $c(i)$ is the scrambling sequence defined by a length-31 Gold sequence [24]. The initialization value of the first sequence is specified with a unit impulse function of length-31. The second scrambling sequence will be initialized with the seed according to

$$
c_{\text {init }}=n_{\mathrm{RNTI}} \cdot 2^{14}+n_{\mathrm{f}} \bmod 2 \cdot 2^{13}+\left\lfloor\frac{n_{\mathrm{s}}}{2}\right\rfloor \cdot 2^{9}+N_{\mathrm{ID}}^{\mathrm{Ncell}}
$$

where $n_{\mathrm{RNTI}}$ denotes the index of the radio network temporary identifier (RNTI), $n_{\mathrm{s}}$ is the first slot of the transmission of the codeword, and the narrowband cell identity number can be selected from the set $N_{\mathrm{ID}}^{\mathrm{Ncell}} \in\{0,1, \cdots, 503\}$. The scrambling sequence will be reinitialized for the repetitions of NPUSCH according to (6) after every $N_{\text {identical }}^{\mathrm{NPUSCH}}$ transmission with $n_{\mathrm{s}}$ and $n_{\mathrm{f}}$ set to the first slot and the frame, respectively. In constellation mapping of NPUSCH transmission, the block of bits $\widetilde{b}(i)$ is modulated by employing low PAPR modulation schemes (e.g., $\pi / 2$-BPSK and $\pi / 4$ QPSK), which are specified for NB-IoT systems to improve the power efficiency at the transmitter (i.e., NB-IoT UE). Thus, we have a block of complex-valued modulation symbols $\boldsymbol{s}=\left[s(0), s(1), \ldots, s\left(N_{\text {symb }}-1\right)\right]^{T}$, where $N_{\text {symb }}$ denotes the number of modulated symbols.

The block of modulation symbols $s$ is divided into $N_{\text {symb }} /$ $M_{\text {sc }}^{\mathrm{NPUSCH}}$ sets, each corresponding to one SC-FDMA symbol. The parameter $M_{\mathrm{sc}}^{\mathrm{NPUSCH}}=N_{\mathrm{RB}}^{\mathrm{NPUSCH}} \cdot N_{\mathrm{sc}}^{\mathrm{RB}}$ indicates the number of subcarriers allocated for NPUSCH transmission, where $N_{\mathrm{RB}}^{\mathrm{NPUSCH}}$ (e.g., $N_{\mathrm{RB}}^{\mathrm{NPUSCH}}=1$ for NB-IoT) corresponding to the bandwidth of NPUSCH in terms of PRB and $N_{s c}^{\mathrm{RB}}$ is the number of subcarriers in a PRB. The frequency-domain symbols after performing DFT operation can be represented as

$$
\begin{aligned}
& S\left(l \cdot M_{\mathrm{sc}}^{\mathrm{NPUSCH}}+k\right)=\frac{1}{\sqrt{M_{\mathrm{sc}}^{\mathrm{NPUSCH}}}} \\
& \cdot \sum_{i=0}^{M_{\mathrm{sc}}^{\mathrm{NPSCH}}-1} s\left(l \cdot M_{\mathrm{sc}}^{\mathrm{NPUSCH}}+i\right) e^{-\mathrm{j} 2 \pi i k / M_{\mathrm{sc}}^{\mathrm{NPSCH}}}, \\
& 0 \leq k \leq M_{\mathrm{sc}}^{\mathrm{NPUSCH}}-1,0 \leq l \leq N_{\mathrm{symb}} / M_{\mathrm{sc}}^{\mathrm{NPUSCH}}-1 .
\end{aligned}
$$

The physical resource element mapping is accomplished by placing frequency-domain user data symbols and known NDMRS symbols within the uplink time-frequency grid. NPUSCH can be mapped to one or more than one RU according to [25], each of which can be transmitted $M$ times. The block of frequency-domain symbols is mapped in a sequential manner (i.e., localized mapping) to subcarriers assigned for transmission [24, 26, 31, 32]. The mapping to resource elements $(k, l)$ corresponding to subcarriers allocated for transmission within a RU will be in increasing order of the first subcarrier index $k$, then the symbol index $l$, and finally the slot number. After mapping to $N_{\text {slots }}$ slots, $N_{\text {slots }}$ repeats $N_{\text {identical }}^{\mathrm{NPUSCH}}$ additional times, before continuing 


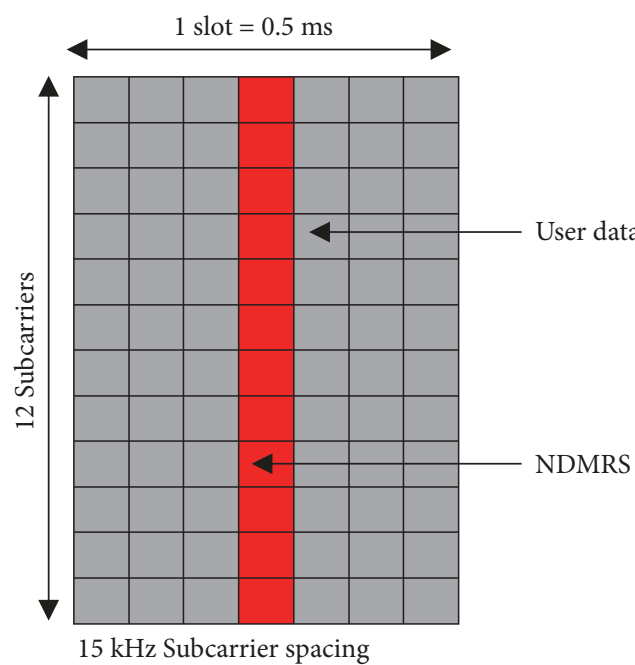

(a)

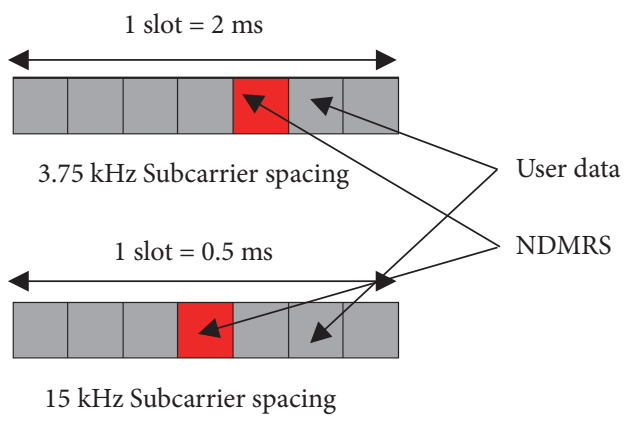

(b)

FIGURE 5: Resource grid mapping for (a) multi-tone (e.g., 12 tone) with $15 \mathrm{kHz}$ subcarrier spacing, and (b) single-tone with both $15 \mathrm{kHz}$ and $3.75 \mathrm{kHz}$ subcarrier spacing.

the mapping of $S(\cdot)$ to the following slot, where the quantities $N_{\text {identical }}^{\mathrm{NPUSCH}}$ and $N_{\text {slots }}$ can be defined as follows:

$$
N_{\text {identical }}^{\mathrm{NPUSCH}}= \begin{cases}\min \left(\left\lceil\frac{M}{2}\right], 4\right) & \text { for } N_{\mathrm{sc}}^{\mathrm{RU}}>1 \\ 1 & \text { for } N_{\mathrm{sc}}^{\mathrm{RU}}=1\end{cases}
$$

and

$$
N_{\text {slots }}= \begin{cases}1 & \Delta f=3.75 \mathrm{kHz} \\ 2 & \Delta f=15 \mathrm{kHz}\end{cases}
$$

where $\Delta f$ denotes the subcarrier spacing. The mapping of $\boldsymbol{S}(\cdot)$ is then repeated until $M N_{\mathrm{RU}} N_{\text {slots }}^{\mathrm{RU}}$ slots have been transmitted. Figure 5 shows the mapping pattern of user data (NPUSCH) symbols and NDMRS symbols within a resource grid for NPUSCH format-1; for example, a RU contains 12 subcarriers for multi-tone transmission and only one subcarrier for single-tone transmission.

The physical resource element mapping is followed by an inverse DFT (IDFT) operation to convert the data into time-domain signal. For single-tone transmission, the timedomain baseband signal $x_{k, l}(t)$ after the CP insertion with length $N_{\mathrm{CP}, l}$ and PS operation for the $k$-th subcarrier in SCFDMA symbol $l$ in an uplink slot can be expressed as

$$
\begin{aligned}
& x_{k, l}(t)=A_{k^{(-)}, l} \cdot e^{j \varphi_{k, l}} \cdot e^{j 2 \pi(k+1 / 2) \Delta f\left(t-N_{\mathrm{CP}, l} T_{\mathrm{s}}\right)}, \\
& k^{(-)}=k+\left\lfloor\frac{N_{\mathrm{sc}}^{\mathrm{RU}}}{2}\right\rfloor
\end{aligned}
$$

for $0 \leq t<\left(N_{\mathrm{CP}, l}+N\right) T_{\mathrm{s}}$, where parameters for $\Delta f=15 \mathrm{kHz}$ and $\Delta f=3.75 \mathrm{kHz}$ are specified in Table $3, A_{k^{(-)}, l}$ is the frequency-domain modulation value of symbol $l$, and the phase rotation $\varphi_{k, l}$ is defined as [24]

$$
\begin{aligned}
& \varphi_{k, l}=\rho(\widetilde{l} \bmod 2)+\widehat{\varphi}_{k}(\widetilde{l}) \\
& \rho= \begin{cases}\frac{\pi}{2} & \text { for BPSK } \\
\frac{\pi}{4} & \text { for QPSK }\end{cases} \\
& \widehat{\varphi}_{k}(\tilde{l}) \\
& = \begin{cases}0 & \tilde{l}=0 \\
\widehat{\varphi}_{k}(\widetilde{l}-1)+2 \pi \Delta f\left(k+\frac{1}{2}\right)\left(N+N_{\mathrm{CP}, l}\right) T_{\mathrm{s}} & \widetilde{l}>0\end{cases} \\
& \tilde{l}=0,1, \ldots, M N_{\mathrm{RU}} N_{\text {slots }}^{\mathrm{RU}} N_{\text {symb }}^{\mathrm{RU}}-1, l=\tilde{l} \bmod N_{\text {symb }}^{\mathrm{RU}}
\end{aligned}
$$

where $\widetilde{l}$ is the symbol counter that is reset at the start of a transmission and incremented for each symbol during the time of transmission.

The time-domain signal $x_{l}(t)$ in SC-FDMA symbol $l$ in an uplink slot for multi-tone transmission can be modelled as

$$
x_{l}(t)=\sum_{k=-\left\lfloor N_{\mathrm{sc}}^{\mathrm{RU}} / 2\right\rfloor}^{\left\lceil N_{\mathrm{sc}}^{\mathrm{RU}} / 2\right\rceil-1} A_{k^{(-)}, l} \cdot e^{j 2 \pi(k+1 / 2) \Delta f\left(t-N_{\mathrm{CP}, l} T_{\mathrm{s}}\right)}
$$

for $0 \leq t<\left(N_{\mathrm{CP}, l}+N\right) \times T_{\mathrm{s}}$, where $k^{(-)}=k+\left\lfloor N_{\mathrm{sc}}^{\mathrm{RU}} / 2\right\rfloor$, $N=2048, \Delta f=15 \mathrm{kHz}$, and $A_{k^{(-)}, l}$ is the content of resource element $(k, l)$. Note that only normal CP length $N_{\mathrm{CP}, l}$ of existing LTE is supported in release-13 of the NB-IoT specification.

The time-domain baseband signal is upconverted by a RF front-end and then transmits through a multipath fading channel whose delay speared is assumed to be smaller than the CP length. The received signal is composed of 
TABLE 3: SC-FDMA parameters for $N_{\mathrm{sc}}^{\mathrm{RU}}=1$.

\begin{tabular}{lcc}
\hline Parameter & \multicolumn{2}{c}{ Subcarrier spacing } \\
\hline$N$ & $\mathbf{3 . 7 5} \mathbf{k H z}$ & $\mathbf{1 5} \mathbf{k H z}$ \\
\hline Cyclic prefix length $N_{\mathrm{CP}, l}$ & 8192 & 160 for $l=0$ \\
\hline Set of values for $k$ & 256 & 144 for $l=1,2, \ldots, 6$ \\
\hline
\end{tabular}

the signals from different channel paths and additive noise; then resultant signal for both single-tone and multi-tone transmissions can be represented as the circular convolution of transmitted signal and channel impulse response (CIR). Thus, we have

$$
\begin{aligned}
& y_{\text {single }}(t)=x_{k, l}(t) \otimes h(t)+n(t) \\
& y_{\text {multi }}(t)=x_{l}(t) \otimes h(t)+n(t)
\end{aligned}
$$

where $n(t)$ is the additive white Gaussian noise (AWGN) with zero mean and variance $\sigma_{n}{ }^{2}, y_{\text {single }}(t)$, and $y_{\text {multi }}(t)$ are the received signal for single-tone and multi-tone transmissions, respectively, and $h(t)$ denotes the CIR of the multipath fading channel with $L$ distinct complex-taps, which can be expressed as

$$
h(t)=\sum_{i=0}^{L-1} \beta_{i} \delta\left(t-\tau_{i}\right)
$$

where $\beta_{i}$ and $\tau_{i}$ represent the attenuation and the delay of the $i$-th path, respectively. Therefore, the noisy and delayed version of the signals at the receiver can be written as

$$
\begin{aligned}
y_{\text {single }}(t) & =\sum_{i=0}^{L-1} \beta_{i} x_{k, l}\left(t-\tau_{i}\right)+n(t) . \\
y_{\text {multi }}(t) & =\sum_{i=0}^{L-1} \beta_{i} x_{l}\left(t-\tau_{i}\right)+n(t) .
\end{aligned}
$$

After removing $\mathrm{CP}$, the receiver performs inverse operations of the NPUSCH and UL-SCH processing. In addition, NDMRS-assisted frequency-domain channel estimation and equalization are performed.

\section{Channel Estimation in NB-IoT Uplink}

4.1. Theoretical Analysis. We first compute the channel estimates for all the allocated subcarriers in a RU of the symbols (i.e., $l=3,10$ or 4,11 , depending on the subcarrier spacing) within a subframe that contain NDMRS sequences. Then, we obtain the channel estimates for the rest of the symbols employing one dimensional (1D) time-domain interpolation of the channel estimates within one subframe of a RU. NPUSCH and NDMRS hopping are not considered in this work to make out derivations generally applicable to any multicarrier communication systems. The NDMRS-aided channel estimation can be done by using widely used estimation algorithms like LS [33] estimator and MMSE [34] estimator. We assume that all the scheduled number of subcarriers $N_{\mathrm{sc}}^{\mathrm{RU}}$ in a RU are occupied by NDMRS symbols (i.e., pilots) $r_{u}(n)$ generated in Section 3.1 within the specified symbol locations. Then, the group of received pilot symbols $\boldsymbol{R}$ in the frequency-domain can be represented as

$$
\boldsymbol{R}=\left[R(0), R(1), \ldots, R\left(N_{\mathrm{sc}}^{\mathrm{RU}}-1\right)\right]^{T} .
$$

For the pilot symbol $R, \boldsymbol{H}_{R}$ is the true channel frequency response (CFR) at the pilot locations and $\widetilde{\boldsymbol{H}}_{R}$ represents $R \times 1$ Gaussian white noise vector and its noise variance $\sigma_{\widetilde{H}_{R}}^{2}$. Then, CFR estimates $\widehat{\boldsymbol{H}}_{R}$ can be written as

$$
\widehat{\boldsymbol{H}}_{R}=\boldsymbol{H}_{R}+\widetilde{\boldsymbol{H}}_{R}=\boldsymbol{F}_{R} \boldsymbol{H}+\widetilde{\boldsymbol{H}}_{R}
$$

where $\boldsymbol{H}$ is the $L \times 1$ channel coefficient matrix in frequencydomain, $L$ denotes the maximum channel delay speared, which is assumed to be shorter than the NB-IoT supported $\mathrm{CP}$ length $N_{\mathrm{CP}, l}$, and $\boldsymbol{F}_{\mathrm{R}}$ represents $R \times L$ matrix. Therefore, the channel estimates $\widehat{\boldsymbol{H}}_{\text {LS }}^{\text {con. }}$ based on the conventional LS method of the whole channel response can be obtained as

$$
\widehat{\boldsymbol{H}}_{\mathrm{LS}}^{\text {con. }}=\boldsymbol{F}_{L}\left(\boldsymbol{F}_{R}^{H} \boldsymbol{F}_{R}\right)^{-1} \boldsymbol{F}_{R}^{H} \widehat{\boldsymbol{H}}_{R}
$$

where $\boldsymbol{F}_{L}$ is the $N_{\mathrm{sc}}^{\mathrm{RU}} \times L$ matrix which has the lines where NDMRS symbols are located and the previous column of $N_{\mathrm{sc}}^{\mathrm{RU}} \times N_{\mathrm{sc}}^{\mathrm{RU}}$ DFT matrix.

The LS algorithm is computationally less complex but the problem is that the quantity $\left(\boldsymbol{F}_{R}^{H} \boldsymbol{F}_{R}\right)^{-1}$ in (20), which turns out to be an ill-conditioned matrix. Thus, the conventional LS estimator cannot be a practical estimator to NB-IoT uplink systems due to the presence of some subcarriers without SC-FDMA modulation. The problem of conventional LS estimator can be mitigated to fit in the low complexity NBIoT systems by adding a normalization matrix $\eta \boldsymbol{I}_{L}$, where $\eta$ is a regularization parameter and its value has to be chosen from the range $0 \sim 1$ such that the resulting eigenvalues are all defined and the inverse matrix is least perturbed and $\boldsymbol{I}_{L}$ denotes the identity matrix. Therefore, the channel estimates $\widehat{\boldsymbol{H}}_{\mathrm{LS}}^{\text {prop. }}$ of the proposed LS estimator in frequency-domain can be estimated as

$$
\widehat{\boldsymbol{H}}_{\mathrm{LS}}^{\text {prop. }}=\boldsymbol{F}_{L}\left(\boldsymbol{F}_{R}^{H} \boldsymbol{F}_{R}+\eta \boldsymbol{I}_{L}\right)^{-1} \boldsymbol{F}_{R}^{H} \widehat{\boldsymbol{H}}_{R} .
$$

The mean square error (MSE) $\varepsilon_{\mathrm{LS}}^{\text {prop. }}$ of the proposed LS estimator can be computed as

$$
\varepsilon_{\mathrm{LS}}^{\text {prop. }}=\mathrm{E}\left[\left\|\widehat{\boldsymbol{H}}_{\mathrm{LS}}^{\text {prop. }}-\boldsymbol{H}\right\|^{2}\right] .
$$


Consequently, after simplification of (22), we have

$$
\varepsilon_{\mathrm{LS}}^{\text {prop. }}=\sigma_{\widetilde{H}_{R}}^{2} \boldsymbol{F}_{L}\left(\boldsymbol{F}_{R}^{H} \boldsymbol{F}_{R}+\eta \boldsymbol{I}_{L}\right)^{-1} \boldsymbol{F}_{R}^{H} .
$$

The MMSE is an optimal estimation technique that exploits the knowledge of the channel statistics and channel covariance matrix. For the conventional MMSE estimator, we have

$$
\widehat{\boldsymbol{H}}_{\mathrm{MMSE}}^{\text {con. }}=\boldsymbol{F}_{L}\left(\boldsymbol{F}_{R}^{H} \boldsymbol{F}_{R}+\sigma_{\widetilde{H}_{R}}^{2} \boldsymbol{\Lambda}^{-1}\right)^{-1} \boldsymbol{F}_{R}^{H} \widehat{\boldsymbol{H}}_{R}
$$

where $\boldsymbol{\Lambda}=\mathrm{E}\left[\boldsymbol{H} \boldsymbol{H}^{H}\right]$ represents the autocovariance matrix of $\boldsymbol{H}$. MMSE is a modified form of conventional LS estimator in (20), but it is very intricate to obtain the precise knowledge of the channel covariance matrix in very low SNR regime. For the application of MMSE in NB-IoT uplink systems, we assume that the delay spectrum of the channel power is evenly distributed, then the channel covariance matrix $\Lambda$ turns out to be an identity matrix $\boldsymbol{I}_{L}$, resulting in the elimination of real time matrix inversion. Furthermore, the noise power is also normalized by dividing the average power $\sigma_{R}^{2}$ of the NDMRS symbols. Thus, channel estimates $\widehat{\boldsymbol{H}}_{\mathrm{MMSE}}^{\text {prop }}$ for the proposed MMSE estimator can be estimated as

$$
\widehat{\boldsymbol{H}}_{\mathrm{MMSE}}^{\text {prop. }}=\boldsymbol{F}_{L}\left[\boldsymbol{F}_{R}^{H} \boldsymbol{F}_{R}+\left(\frac{\sigma_{\widetilde{H}_{R}}^{2}}{\sigma_{R}^{2}}\right) \boldsymbol{I}_{L}\right]^{-1} \boldsymbol{F}_{R}^{H} \widehat{\boldsymbol{H}}_{R} .
$$

The MSE of the proposed method $\varepsilon_{\text {MMSE }}^{\text {prop. }}$ can be computed as

$$
\varepsilon_{\mathrm{MMSE}}^{\text {prop. }}=\mathrm{E}\left[\left\|\widehat{\boldsymbol{H}}_{\mathrm{MMSE}}^{\text {prop. }}-\boldsymbol{H}\right\|^{2}\right] \text {. }
$$

Subsequently, the simplified form of (26) can be represented as the following form:

$$
\varepsilon_{\mathrm{MMSE}}^{\text {prop. }}=\left\|\Lambda-\Lambda\left(1+\frac{\Gamma}{\Upsilon}\left(\Lambda^{-1}\right)\right)^{-1}\right\|
$$

where $\Upsilon$ represents the average SNR, which is defined as

$$
\Upsilon=\frac{\sigma_{R}^{2}}{\sigma_{\widetilde{H}_{R}}^{2}} .
$$

and

$$
\Gamma=\mathrm{E}\left[\left|R\left(N_{\mathrm{sc}}^{\mathrm{RU}}\right)\right|^{2}\right] \mathrm{E}\left[\left|\frac{1}{R\left(N_{\mathrm{sc}}^{\mathrm{RU}}\right)}\right|^{2}\right]
$$

where $\Gamma$ is the modulation scheme dependent constant, for example, $\Gamma=1$ for QPSK modulation.

4.2. Simulation Results and Analysis. We have considered LTE-based NB-IoT uplink systems whose parameters are selected based on the specifications of 3GPP NB-IoT in release-13. We have investigated and compared the performance of our proposed NDMRS-assisted channel estimation algorithms with conventional LS and MMSE algorithms in terms of BER in contrast to SNR. In this paper, we have considered a simple single-input single-output (SISO) system
TABLE 4: Simulation parameters.

\begin{tabular}{lc}
\hline Parameter & Value \\
\hline System bandwidth & $180 \mathrm{kHz}$ \\
Carrier bandwidth & $900 \mathrm{MHz}$ \\
Subcarrier spacing & $15 \mathrm{kHz}$ and $3.75 \mathrm{kHz}$ \\
Transmission mode & Singe-tone and multi-tone $(3,6$ or 12$)$ \\
Channel coding & Turbo $(1 / 3$-coding rate $)$ \\
Modulation schemes & BPSK and QPSK \\
CRC & 24 bits \\
Antenna configuration & SISO $(1 \mathrm{Tx} \times 1 \mathrm{Rx})$ \\
Propagation channel & Typical urban $(\mathrm{TU}), f_{\mathrm{d}}=1 \mathrm{~Hz}$ \\
Channel estimation & Modified LS and MMSE \\
Channel equalization & Zero forcing $(\mathrm{ZF})$ \\
Number of iterations & $10^{5}$ \\
\hline
\end{tabular}

for both single-tone transmission with $15 \mathrm{kHz}$ and $3.75 \mathrm{kHz}$ subcarrier spacing and multi-tone transmission with $15 \mathrm{kHz}$ subcarrier spacing. We have set the repetition number to guarantee the transmission reliability (i.e., $\mathrm{BER}<10^{-1}$ ) at low SNR. Transmission time and resource utilization are also our concern because low transmission time and high rate of resource utilization can improve the data rate of NBIoT systems. Low complexity zero forcing $(\mathrm{ZF})$ equalizer is employed. In this simulation, we have considered identical transmission time and resource utilization. The fundamental parameters are used to carry out simulations as listed in Table 4 and referred to figure captions for better readability.

Simulation results of the performance of singletone transmission for different channel estimators using $\pi / 2-$ BPSK modulation are shown in Figure 6. It is observed that the channel estimation accuracy cannot be improved when SNR is extremely low, but estimation precision rises as the receive SNR increases (i.e., better channel condition). For $15 \mathrm{kHz}$ subcarrier spacing as shown in Figure 6(a), our proposed LS and MMSE estimators perform better than the traditional LS and MMSE estimators. As shown in Figure 6(b), the system performance of $3.75 \mathrm{kHz}$ subcarrier spacing employing $\pi / 2-\mathrm{BPSK}$ for all estimation methods is slightly lower compared to $15 \mathrm{kHz}$ subcarrier spacing.

The BER performance curves of different channel estimators employing $\pi / 4$-QPSK constellation for single-tone transmission are shown in Figure 7 . The simulation results elucidate that the system performance with $\pi / 4$-QPSK modulation is little bit lower than $\pi / 2$-BPSK modulation due to extremely low SNR values. However, the system performance improves with our proposed algorithms compared to the conventional LS and MMSE algorithms regardless of the modulation scheme and subcarrier spacing.

The BER performance curves of NPUSCH format-1 for multi-tone (e.g., 12-tone) transmission for different channel estimation techniques are shown in Figure 8. It is also seen that the system performs better with our proposed algorithms than the traditional LS and MMSE algorithms. Since NBIoT supports only phase-shift-keying (PSK) modulation, the receiver's performance of such two algorithms has linear change and no significant variation when SNR is extremely 


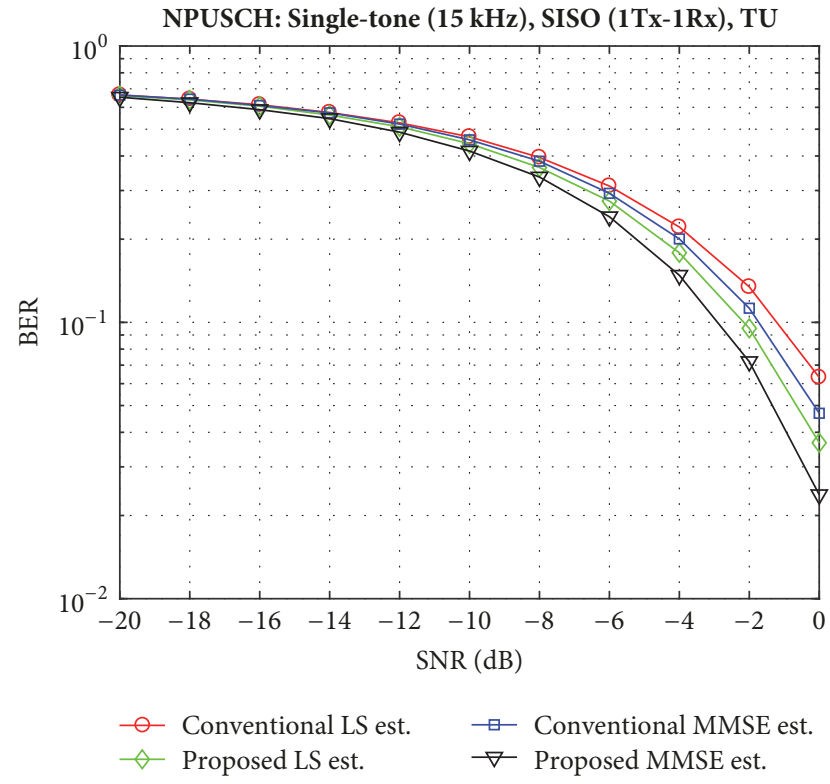

(a)

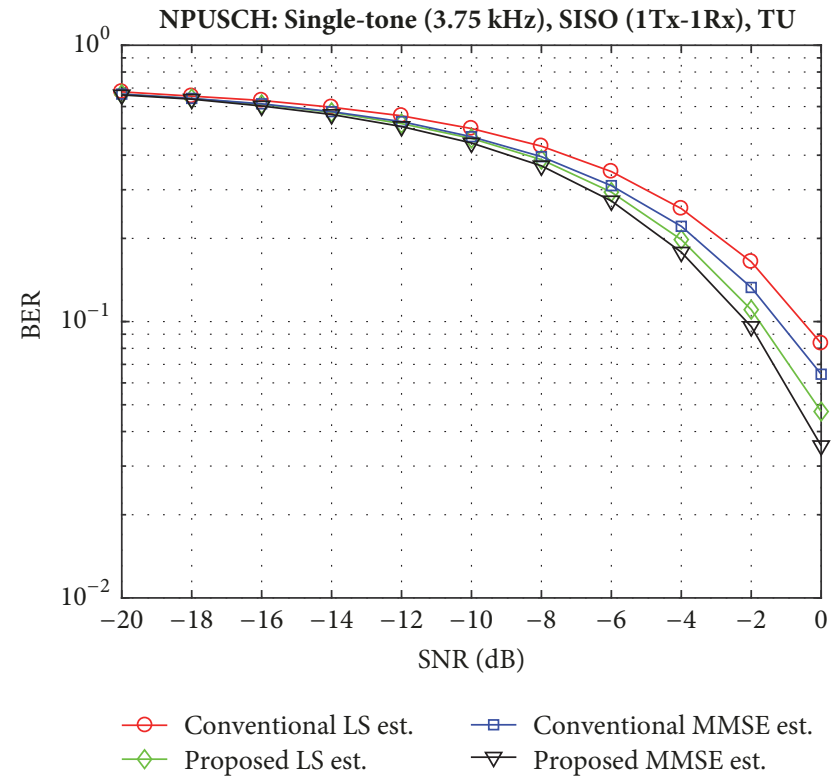

(b)

FIGURE 6: BER performance of NPUSCH for single-tone transmission with $\pi / 2-$ BPSK modulation, when $\mathrm{MCS}=0$, RU $=1$, TBS $=16$, and the transmission time is $512 \mathrm{~ms}$ : (a) $15 \mathrm{kHz}$ subcarrier spacing with repetitions $M=64$, and (b) $3.75 \mathrm{kHz}$ subcarrier spacing with repetitions $M=16$.

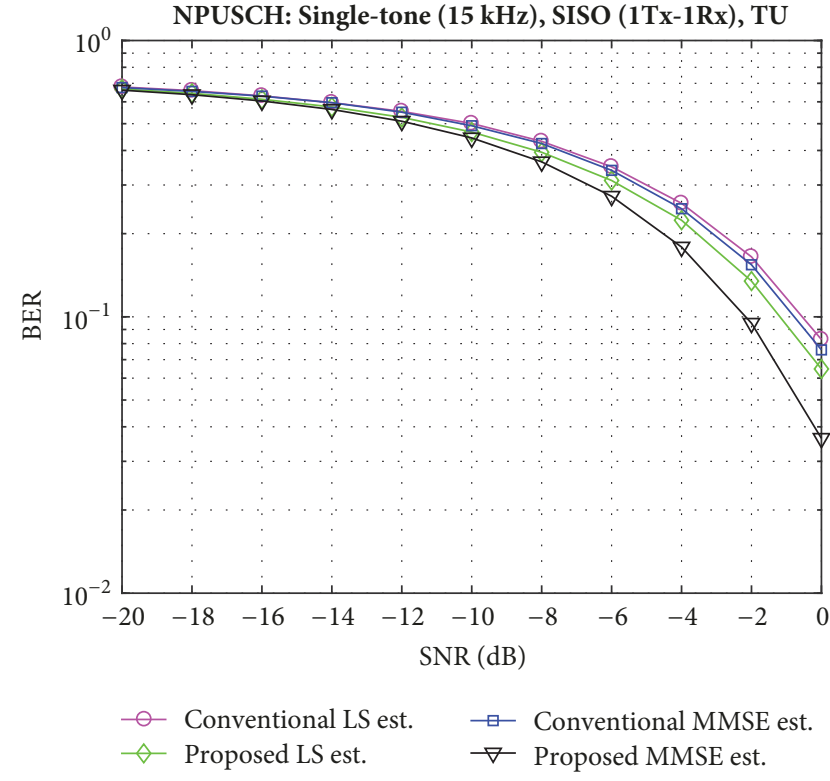

(a)

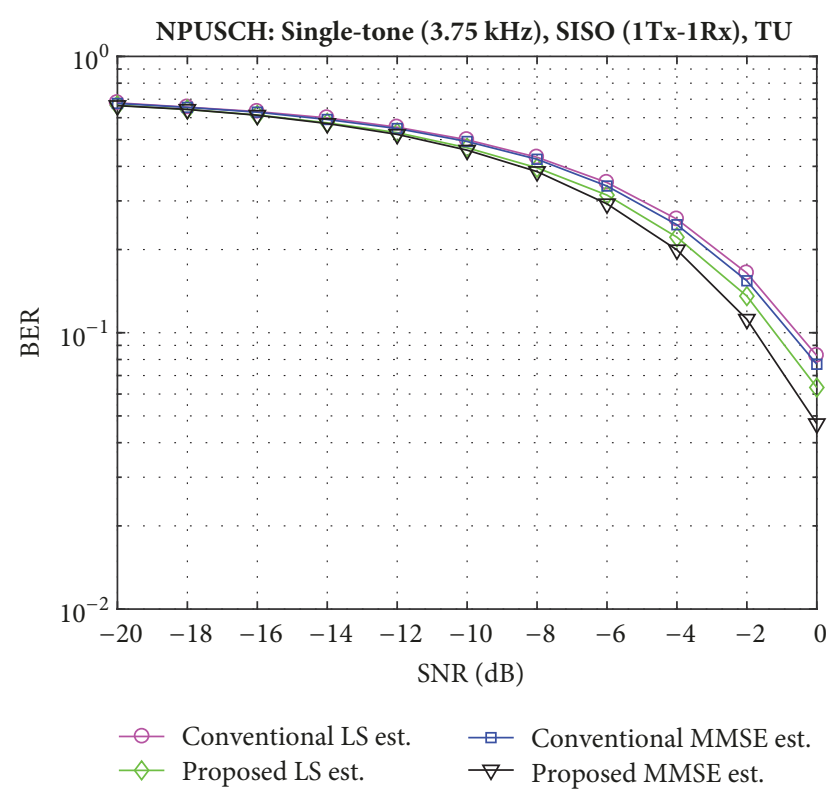

(b)

FIGURE 7: BER performance of NPUSCH for single-tone transmission with $\pi / 4-$ QPSK modulation, when $\mathrm{MCS}=4$, RU $=1$, TBS $=56$, and the transmission time is $512 \mathrm{~ms}$ : (a) $15 \mathrm{kHz}$ subcarrier spacing with repetitions $M=64$, and (b) $3.75 \mathrm{kHz}$ subcarrier spacing with repetitions $M=16$. 


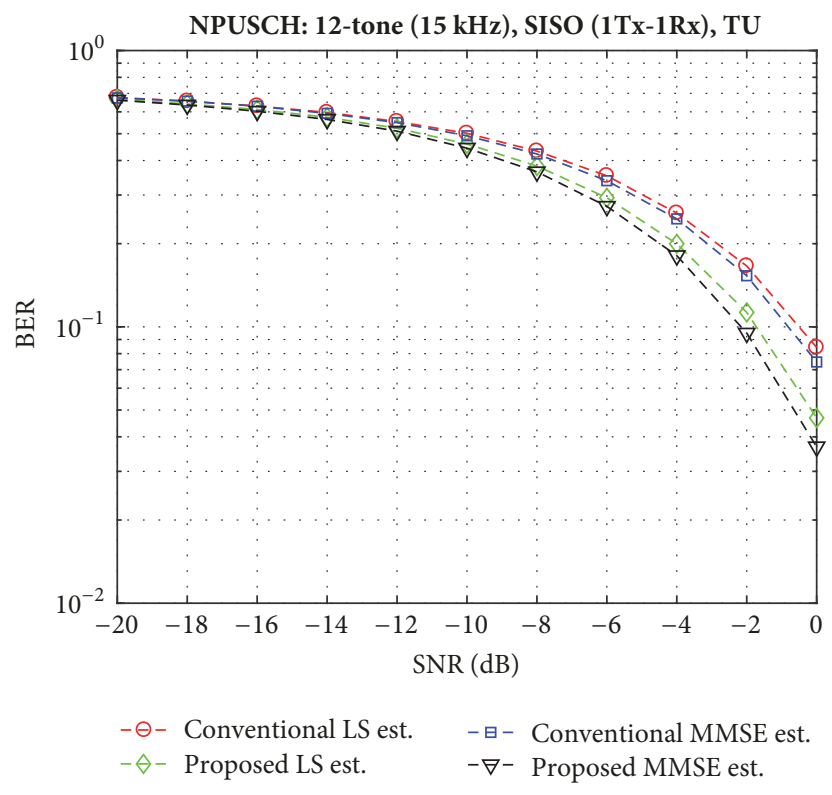

FIGURE 8: BER performance of NPUSCH for multi-tone (e.g., 12tone) transmission with $15 \mathrm{kHz}$ subcarrier spacing using QPSK modulation, when $\mathrm{MCS}=4, \mathrm{RU}=8, \mathrm{TBS}=552$, and repetitions $M=64$. The transmission time is $512 \mathrm{~ms}$.

lower. Finally, we conclude that our proposed MMSE algorithm can be coped with the practical implementation of NBIoT uplink systems to ensure successful transmission of user data for both single-tone and multi-tone transmissions.

\section{PAPR Analysis of NB-IoT Uplink}

5.1. Theoretical Analysis. The baseband time-domain transmit signals $x_{k, l}(t)$ and $x_{l}(t)$ are derived in (10) and (12) for

single-tone and multi-tone transmissions, respectively. To make our derivations generally applicable to any multicarrier communication systems, we assume that $x(t)$ is the continuous time baseband SC-FDMA signal for both types of transmission. The PAPR of the time-domain baseband SCFDMA signal $x(t)$ can be defined as the ratio of the maximum instantaneous power $P_{\max }$ (i.e., peak power) to the average power $P_{\text {avg }}$ of the signal. Thus, we have

$$
\operatorname{PAPR}[x(t)]=\frac{P_{\max }}{P_{\mathrm{avg}}}
$$

where

$$
P_{\max }=\max _{0 \leq t \leq N_{\mathrm{sc}}^{\mathrm{RU}} T_{s}}\left[|x(t)|^{2}\right]
$$

and

$$
P_{\text {avg }}=\frac{1}{N_{\mathrm{sc}}^{\mathrm{RU}}} \int_{0}^{N_{\mathrm{sc}}^{\mathrm{RU}} T_{\mathrm{s}}} \mathrm{E}\left[|x(t)|^{2}\right] d t
$$

where $T_{s}$ is the symbol duration. In NB-IoT uplink transmitter (i.e., NB-IoT UE), the PAPR can be reduced by exploiting linear filtering operation referred to as pulse shaping to limit the out-of-band radiation, which decreases the spectral efficiency. In this paper, RC and RRC filters are employed to pulse shape the SC-FDMA signals. The RC filter can be characterized by the roll-off factor $\delta$ and the symbol duration $T_{s}$. Then, the impulse response of the RC filter in timedomain can be expressed as

$$
h_{\mathrm{RC}}(t)=\frac{\sin \left(\pi t / T_{s}\right) \cdot \cos \left(\pi \delta t / T_{s}\right)}{\left(\pi t / T_{s}\right)\left(1-4 \delta^{2} t^{2} / T_{s}^{2}\right)} .
$$

Equation (33) can also be expressed in frequency-domain as

$$
H_{\mathrm{RC}}(f)= \begin{cases}T_{s}, & 0 \leq|f| \leq \frac{1-\delta}{2 T_{s}} \\ \frac{T_{s}}{2}\left\{1+\cos \left[\frac{\pi T_{s}}{\delta}\left(|f|-\frac{1-\delta}{2 T_{s}}\right)\right]\right\}, & \frac{1-\delta}{2 T_{s}} \leq|f| \leq \frac{1+\delta}{2 T_{s}} \\ 0, & |f| \geq \frac{1+\delta}{2 T_{s}}\end{cases}
$$

The square-root of the RC filter output characterizes the impulse response of the RRC filter. Therefore, the impulse response of the RRC filter in frequency-domain can be written as

$$
H_{\mathrm{RRC}}(f)=\sqrt{H_{\mathrm{RC}}(f)} .
$$

Consequently, the channel impulse response of RRC filter in time-domain can be represented as

$$
\begin{aligned}
& h_{\mathrm{RRC}}(t) \\
& =\frac{\sin \left\{\left(\pi t / T_{s}\right)(1-\delta)\right\}+\left(4 \delta t / T_{s}\right) \cos \left\{\left(\pi t / T_{s}\right)(1+\delta)\right\}}{\left(\pi t / T_{s}\right)\left(1-16 \delta^{2} t^{2} / T_{s}^{2}\right)} .
\end{aligned}
$$

Finally, the distribution of PAPR of the baseband SC-FDMA signal $x(t)$ is the most practical performance indicator. D. Wulich et al. in [35] have investigated the amplitude of a single-carrier modulated signal that does not have a Gaussian distribution and it is also hard to deduce analytically the precise form of the distribution. In this paper, we perform numerical analysis to investigate the PAPR properties of SCFDMA signals. For a given threshold value of PAPR $\psi_{0}$, the cumulative distribution function (CDF) can be defined as

$$
F_{\psi}\left(\psi_{0}\right)=\operatorname{Pr}\left(\psi \leq \psi_{0}\right)
$$


TABLE 5: 99.9 percentile PAPR for single-tone transmission.

\begin{tabular}{|c|c|c|c|c|}
\hline \multirow{2}{*}{ Modulation } & \multirow{2}{*}{ Subcarrier spacing $(\mathbf{k H z})$} & \multicolumn{3}{|c|}{ CCDF of PAPR (dB) } \\
\hline & & No PS & RC & RRC \\
\hline \multirow{2}{*}{$\pi / 2$-BPSK } & 15 & 3.64 & 2.74 & 2.34 \\
\hline & 3.75 & 3.55 & 2.46 & 2.25 \\
\hline \multirow{2}{*}{$\pi / 4-\mathrm{QPSK}$} & 15 & 4.40 & 3.50 & 2.75 \\
\hline & 3.75 & 3.70 & 3.45 & 2.70 \\
\hline
\end{tabular}

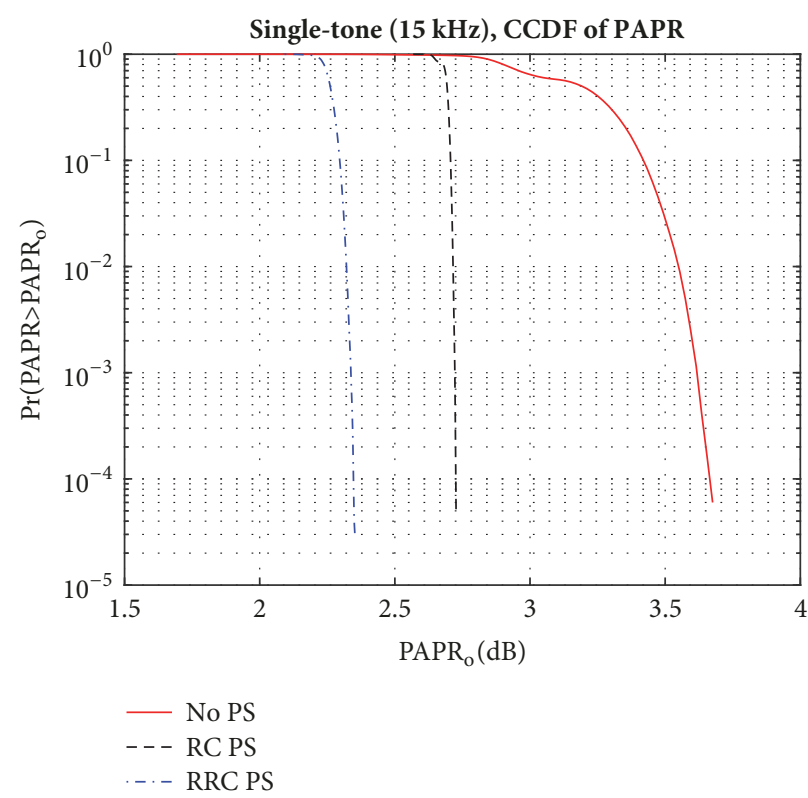

(a)

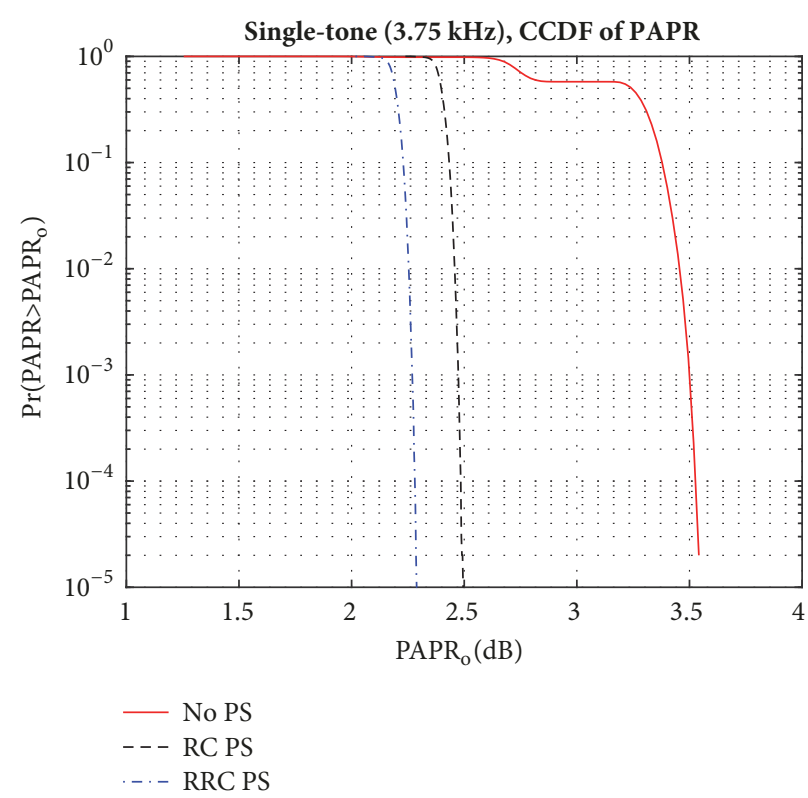

(b)

FIGURE 9: Comparison of CCDF of PAPR for NB-IoT uplink single-tone transmission with and without pulse shaping using $\pi / 2$-BPSK modulation, when TBS $=16$ and roll-off factor $\delta=0.2$ : (a) $15 \mathrm{kHz}$ subcarrier spacing, and (b) $3.75 \mathrm{kHz}$ subcarrier spacing.

where $\psi=\operatorname{PAPR}[x(t)]$. The complementary CDF (CCDF) of PAPR is the probability that the PAPR of the SC-FDMA signal exceeds a given threshold $\psi_{0}$, which can then be expressed as

$$
\operatorname{Pr}\left(\psi \geq \psi_{0}\right)=1-F_{\psi}\left(\psi_{0}\right) .
$$

5.2. Simulation Results and Analysis. The CCDF is taken to represent the statistical probability that the PAPR value of a TBS exceeds a predefined threshold $\mathrm{PAPR}_{0}$. We have considered an NB-IoT uplink transmission system for both single-tone and multi-tone transmissions with $180 \mathrm{kHz}$ system bandwidth. Low PAPR modulation schemes like $\pi / 2$ BPSK and $\pi / 4$-QPSK for single-tone and only QPSK for multi-tone transmissions are employed. Total $10^{5}$ repetitions are employed to calculate the CCDF of PAPR. In addition, the $\mathrm{RC}$ and RRC pulse shaping filters with roll-off factor $\delta=0.2$ and oversampling factor of 4 are used as transmit filter to limit the out-of-band radiation. We have compared the PAPR value that is exceeded with the probability less than 0.1 percent (i.e., $\operatorname{Pr}\left\{\mathrm{PAPR}>\mathrm{PAPR}_{0}\right\}=10^{-3}$ ) PAPR.

Figure 9 shows the comparison of CCDF of PAPR among no pulse shaping, RC, and RRC pulse shaping for single-tone transmission with $\pi / 2$-BPSK modulation. In this case, both
$15 \mathrm{kHz}$ and $3.75 \mathrm{kHz}$ subcarrier spacing types are considered. As shown in Figure 9(a), it is observed that the 0.1 percent or 99.9 percentile PAPR of $15 \mathrm{kHz}$ subcarrier spacing using RRC filter are approximately 1.3 and $0.4 \mathrm{~dB}$ less compared to the no pulse shaping and the RC filter, respectively. On the other hand, $3.75 \mathrm{kHz}$ subcarrier spacing with RRC filter as depicted in Figure 9(b) shows about 1.3 and $0.21 \mathrm{~dB}$ less PAPR value at 0.1 percent of CCDF than without pulse shaping and RC filter, respectively. Figure 10 shows the comparison of CCDF of PAPR with and without pulse shaping for singletone transmission employing $\pi / 4$-QPSK modulation. It can be seen that the PAPR values for $\pi / 4$-QPSK modulation are higher than the PAPR values evaluated with $\pi / 2$-BPSK modulation in Figure 9 regardless of the subcarrier spacing. The PAPR evaluation results for single-tone transmission can be summarized in Table 5 .

The CCDF of PAPR curves with and without pulse shaping for multi-tone (e.g., 3, 6 and 12-tone) transmission employing $\pi / 4$-QPSK modulation are shown in Figure 11. As shown in Figure 11, the PAPR value is increasing as the number of tones increases at the 99.9 percentile of CCDF. Table 6 shows the summery of our evaluations for multi-tone transmission. Finally, we conclude that the lower 
TABLE 6: 99.9 percentile PAPR for multi-tone transmission.

\begin{tabular}{lcccc}
\hline \multirow{2}{*}{ Modulation } & No. of subcarriers & \multicolumn{2}{c}{ CCDF of PAPR (dB) } & RC \\
& & No PS & 3.70 & 2.80 \\
QPSK & 3 & 4.4 & 3.80 & 3.0 \\
& 6 & 6.40 & 3.90 & 3.40 \\
\hline
\end{tabular}

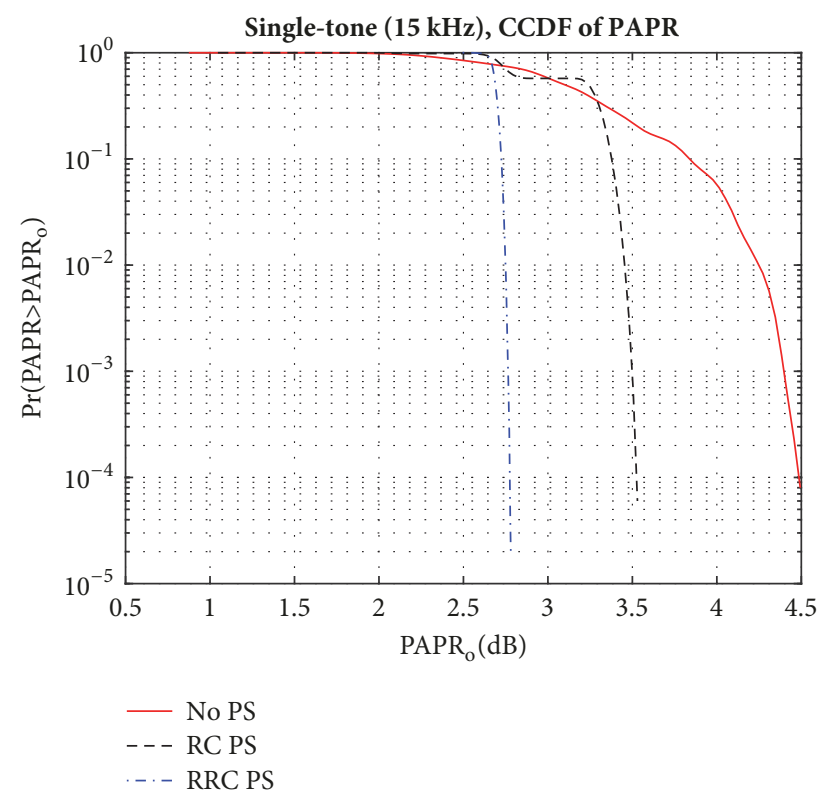

(a)

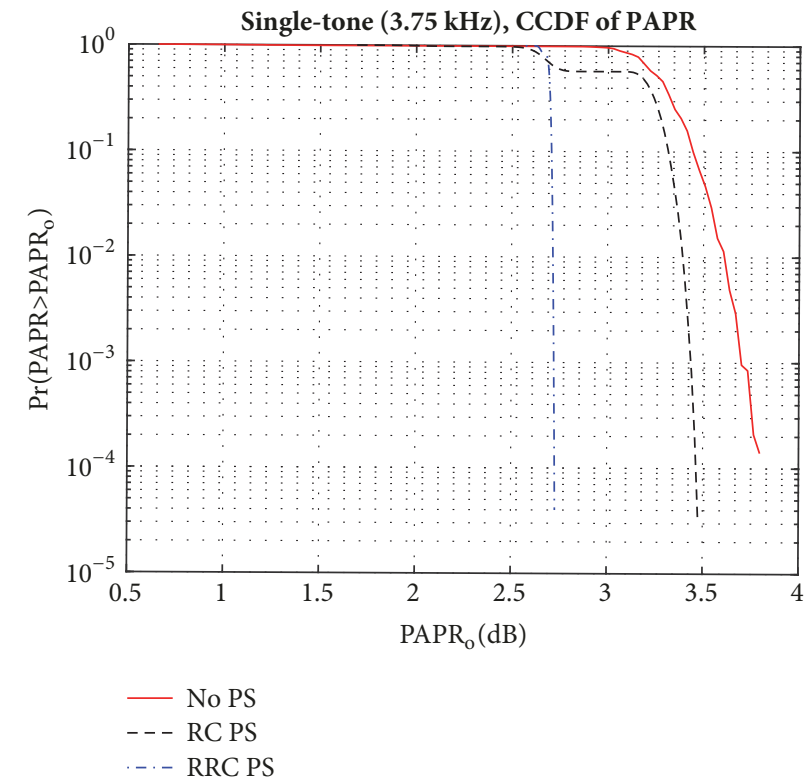

(b)

FIgURE 10: Comparison of CCDF of PAPR for NB-IoT uplink single-tone transmission with and without pulse shaping using $\pi / 4$-QPSK modulation, when TBS $=56$ and roll-off factor $\delta=0.2$ : (a) $15 \mathrm{kHz}$ subcarrier spacing, and (b) $3.75 \mathrm{kHz}$ subcarrier spacing.

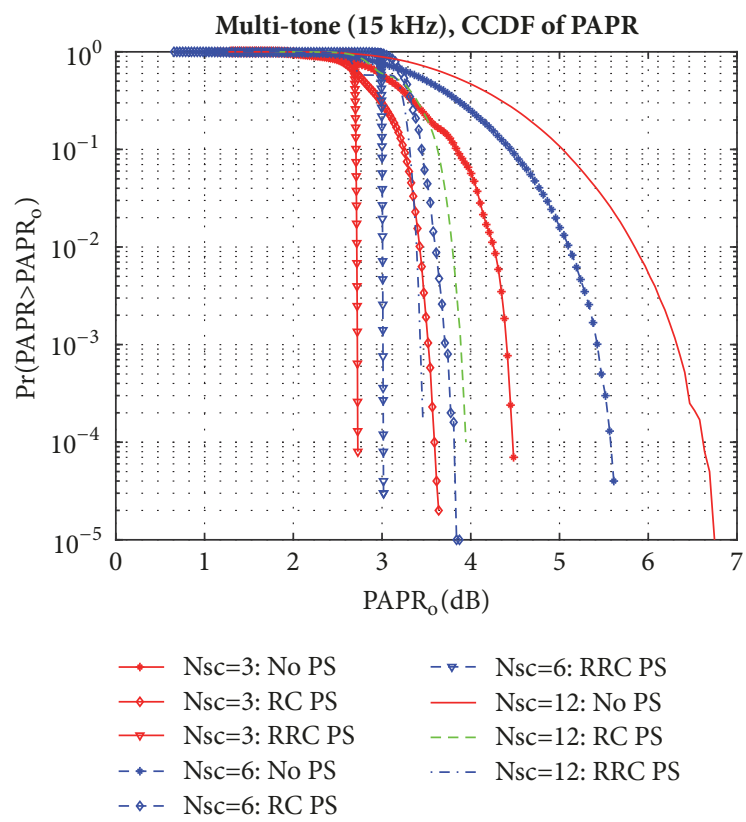

Figure 11: Comparison of CCDF of PAPR for NB-IoT uplink multitone transmission with and without pulse shaping transmit filter using QPSK modulation, when TBS $=56$ and roll-off factor $\delta=0.2$. values of PAPR by using RRC filter is feasible for NB-IoT uplink transmitter, thus requiring very little power back-off to maintain the linearity of the power amplifier.

\section{Conclusion}

In this paper, we have provided a brief survey of NB-IoT technology including deployment options, physical channels and signals, uplink resource grid structure, and resource unit configuration. We have developed a system model for uplink NB-IoT based on the 3GPP specifications in release13. An analytical signal model and NDMRS generation and mapping are presented. To guarantee the successful detection of user data (i.e., $\mathrm{BER}<10^{-1}$ ) in extremely low SNR regime, we have proposed two channel estimation algorithms as a modified form of traditional LS and MMSE estimators. We have investigated the effectiveness of our proposed NDMRSassisted channel estimators compared with others through extensive link-level computer simulations. The simulation results vindicate that our proposed estimation techniques perform better at the $\mathrm{SNR}<0 \mathrm{~dB}$ compared to the conventional LS and MMSE algorithms and suggesting that the proposed algorithms can be adopted to NB-IoT uplink receiver. The improved channel estimation techniques can 
be applied to not only NB-IoT systems, but also in any multicarrier communication systems. Furthermore, we have analyzed and evaluated the PAPR by employing RC and RRC pulse shaping at the transmitter. Through numerical simulations, the PAPR values are evaluated for both singletone and multi-tone transmissions. Our evaluation results show that the RRC pulse shaping with lower PAPR values is feasible to the actual hardware design of low-cost NB-IoT UE. In the future, we will consider carrier frequency offset (CFO) and receiver diversity to improve the system performance in uplink NB-IoT systems.

\section{Conflicts of Interest}

The authors declare that there are no conflicts of interest regarding the publication of this paper.

\section{Acknowledgments}

The authors would like to acknowledge the CAS-TWAS President's Fellowship Program. They would also like to thank the Information Science Laboratory Center of University of Science and Technology of China (USTC) for hardware and software services.

\section{References}

[1] A. Nordrum, "Popular Internet of Things forecast of 50 billion devices by 2020 is outdated," IEEE Spectrum, 2016.

[2] "Cellular networks for massive IoT-enabling low power wide area applications, Ericsson White paper, 2016," https://www. ericsson.com/res/docs/whitepapers/wp_iot.pdf.

[3] A. Diaz-Zayas, C. A. Garcia-Perez, A. M. Recio-Perez, and P. Merino, "3GPP Standards to Deliver LTE Connectivity for IoT," in Proceedings of the 2016 IEEE First International Conference on Internet-of-Things Design and Implementation (IoTDI), pp. 283288, Berlin, Germany, April 2016.

[4] F. Liu, C. Tan, E. T. Lim, and B. Choi, "Traversing knowledge networks: an algorithmic historiography of extant literature on the Internet of Things (IoT)," Journal of Management Analytics, vol. 4, no. 1, pp. 3-34, 2017.

[5] L. D. Xu, W. He, and S. Li, "Internet of things in industries: a survey," IEEE Transactions on Industrial Informatics, vol. 10, no. 4, pp. 2233-2243, 2014.

[6] S. Li, L. D. Xu, and S. Zhao, "The internet of things: a survey," Information Systems Frontiers, vol. 17, no. 2, pp. 243-259, 2015.

[7] R. Want, B. N. Schilit, and S. Jenson, "Enabling the internet of things," The Computer Journal, vol. 48, no. 1, pp. 28-35, 2015.

[8] A. Al-Fuqaha, M. Guizani, M. Mohammadi, M. Aledhari, and M. Ayyash, "Internet of things: a survey on enabling technologies, protocols, and applications," IEEE Communications Surveys \& Tutorials, vol. 17, no. 4, pp. 2347-2376, 2015.

[9] K. Mekki, E. Bajic, F. Chaxel, and F. Meyer, "A comparative study of LPWAN technologies for large-scale IoT deployment," ICT Express, 2018.

[10] J. Petajajarvi, K. Mikhaylov, M. Hamalainen, and J. Iinatti, "Evaluation of LoRa LPWAN technology for remote health and wellbeing monitoring," in Proceedings of the 10th International Symposium on Medical Information and Communication Technology, ISMICT 2016, USA, March 2016.
[11] Introduction of NB-IoT in 36.331, 3GPP RP-161248, 3GPP TSGRAN Meeting 72, Ericsson, Nokia, ZTE, NTT DOCOMO Inc., Busan, South Korea, Jun. 2016.

[12] N. Mangalvedhe, R. Ratasuk, and A. Ghosh, "NB-IoT deployment study for low power wide area cellular IoT," in Proceedings of the 27th IEEE Annual International Symposium on Personal, Indoor, and Mobile Radio Communications, PIMRC 2016, esp, September 2016.

[13] A. Kiayani, L. Anttila, Y. Zou, and M. Valkama, "Channel Estimation and Equalization in Multiuser Uplink OFDMA and SC-FDMA Systems Under Transmitter RF Impairments," IEEE Transactions on Vehicular Technology, vol. 65, no. 1, pp. 82-99, 2016.

[14] J. Xue and S. Li, "An SC-FDMA Channel Estimation Algorithm Research Based on Pilot Signals," in Proceedings of the 2nd International Symposium on Computer, Communication, Control and Automation, China, Feburary 2013.

[15] Y.-P. E. Wang, X. Lin, A. Adhikary et al., "A premier on 3GPP narrowband Internet of Things (NB-IoT)," IEEE Com. Mag, pp. 117-123, 2017.

[16] C. Yu, L. Yu, Y. Wu, Y. He, and Q. Lu, "Uplink scheduling and link adaptation for narrowband internet of things systems," IEEE Access, vol. 5, pp. 1724-1734, 2017.

[17] J. Zou, H. Yu, W. Miao, and C. Jiang, "Packet-Based Preamble Design for Random Access in Massive IoT Communication Systems," IEEE Access, vol. 5, pp. 11759-11767, 2017.

[18] W. Yang, M. Hua, J. Zhang et al., "Enhanced System Acquisition for NB-IoT," IEEE Access, vol. 5, pp. 13179-13191, 2017.

[19] X. Lin, J. Bergman, F. Gunnarsson et al., "Positioning for the Internet of Things: A 3GPP Perspective," IEEE Communications Magazine, vol. 55, no. 12, pp. 179-185, 2017.

[20] S. Hu, A. Berg, X. Li, and F. Rusek, "Improving the Performance of OTDOA Based Positioning in NB-IoT Systems," in Proceedings of the 2017 IEEE Global Communications Conference (GLOBECOM 2017), pp. 1-7, Singapore, December 2017.

[21] Y. D. Beyene, R. Jantti, K. Ruttik, and S. Iraji, "On the performance of narrow-band internet of things (NB-IoT)," in Proceedings of the 2017 IEEE Wireless Communications and Networking Conference, WCNC 2017, USA, March 2017.

[22] L. Zhang, A. Ijaz, P. Xiao, and R. Tafazolli, "Channel Equalization and Interference Analysis for Uplink Narrowband Internet of Things (NB-IoT)," IEEE Communications Letters, vol. 21, no. 10, pp. 2206-2209, 2017.

[23] R. Ratasuk, N. Mangalvedhe, J. Kaikkonen, and M. Robert, "Data Channel Design and Performance for LTE Narrowband IoT," in Proceedings of the 2016 IEEE 84th Vehicular Technology Conference (VTC-Fall), pp. 1-5, Montreal, QC, Canada, September 2016.

[24] Evolved Universal Terrestrial Radio Access (E-UTRA), "Physical channels and modulation," 3GPP Tech. Spec. Group Radio Access Network, V 13.4.0, Rel. 13, Tech. Spec. TS 36.211, 2016, http://www.3gpp.org/ftp/Specs/archive/36_series/36.211/36211d40.zip.

[25] Evolved Universal Terrestrial Radio Access (E-UTRA), "Physical layer procedures," 3GPP Tech. Spec. Group Radio Access Network, V 13.4.0, Rel. 13, Tech. Spec. TS 36.213, 2016, http:// www.3gpp.org/ftp/Specs/archive/36_series/36.213/36213-d40.zip.

[26] Evolved Universal Terrestrial Radio Access (E-UTRA), “User Equipment (UE) Conformance Specification,” Radio Transmission and Reception, 3GPP Tech. Spec. V13.3.0, Rel. 13, Tech. Spec. TS 36.521-1, 2016. 
[27] Evolved Universal Terrestrial Radio Access (E-UTRA), "NBIoT; Technical Report for BS and UE radio transmission and reception," 3GPP Tech. Rep. V 13.0.0, Rel. 13,TR 36.802, 2016.

[28] GSMA., “3GPP Low Power Wide Area Technologies," GSMA White Paper, 2016.

[29] R. Ratasuk, B. Vejlgaard, N. Mangalvedhe, and A. Ghosh, "NBIoT system for M2M communication," in Proceedings of the 2016 IEEE Wireless Communications and Networking Conference Workshops, WCNCW 2016, pp. 428-432, qat, April 2016.

[30] Evolved Universal Terrestrial Radio Access (E-UTRA), "Multiplexing and channel coding," 3GPP Tech. Spec. Group Radio Access Network, V 13.4.0, Rel. 13, Tech. Spec. TS 36.212, 2016, http://www.3gpp.org/ftp/Specs/archive/36_series/36.212/36212d40.zip.

[31] F. E. Abd El-Samie, F. S. Al-kamali, A. Y. Al-Nahari, and M. I. Dessouky, SC-FDMA for Mobile Communications, CRC Press, Boca Raton, FL, USA, 2013.

[32] Evolved Universal Terrestrial Radio Access (E-UTRA), "User Equipment (UE) Radio Transmission and Reception," 3GPP Tech. Spec. V13.1, Rel. 13, Tech. Spec. TS36.101, 2017.

[33] J.-J. van de Beek, O. Edfors, M. Sandell, S. K. Wilson, and P. O. Borjesson, "On channel estimation in OFDM systems," in Proceedings of the 1995 IEEE 45th Vehicular Technology Conference. Part 2 (of 2), pp. 815-819, July 1995.

[34] M. Morelli and U. Mengali, "A comparison of pilot-aided channel estimation methods for OFDM systems," IEEE Transactions on Signal Processing, vol. 49, no. 12, pp. 3065-3073, 2001.

[35] D. Wulich and L. Goldfeld, "Bound of the distribution of instantaneous power in single carrier modulation," IEEE Transactions on Wireless Communications, vol. 4, no. 4, pp. 1773-1778, 2005. 


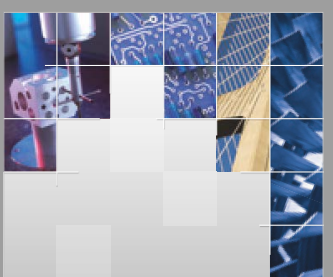

\section{Enfincering}
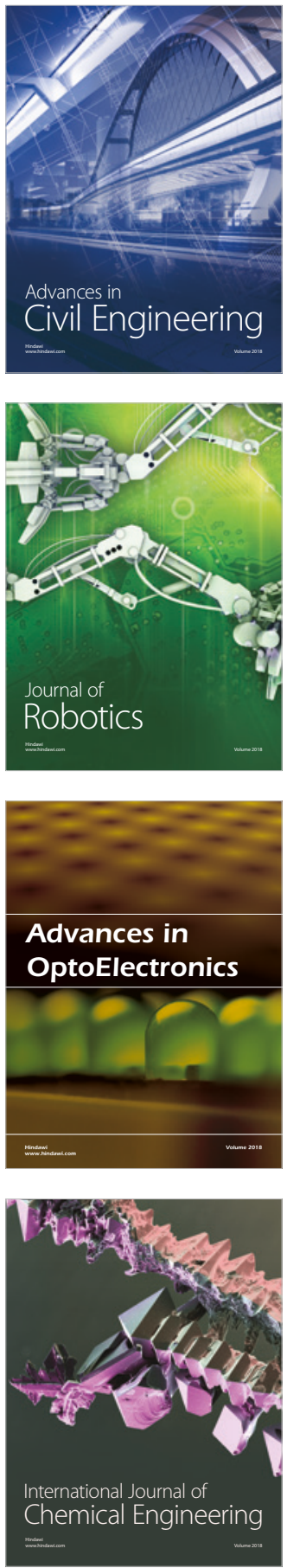

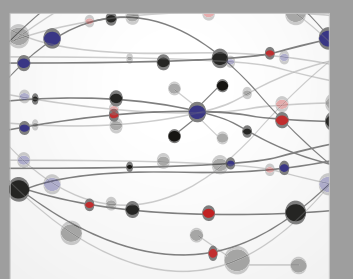

\section{Rotating \\ Machinery}

The Scientific World Journal

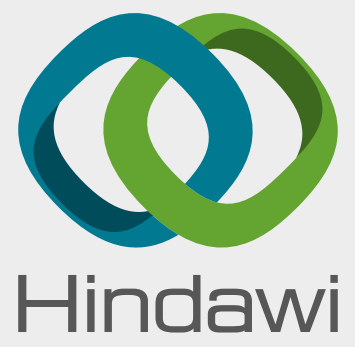

Submit your manuscripts at

www.hindawi.com
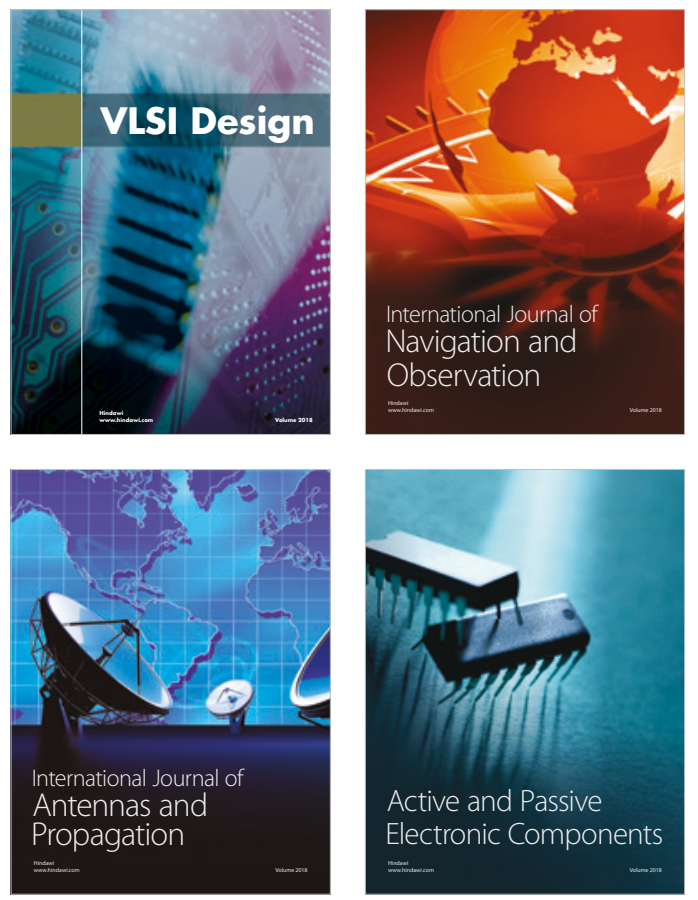
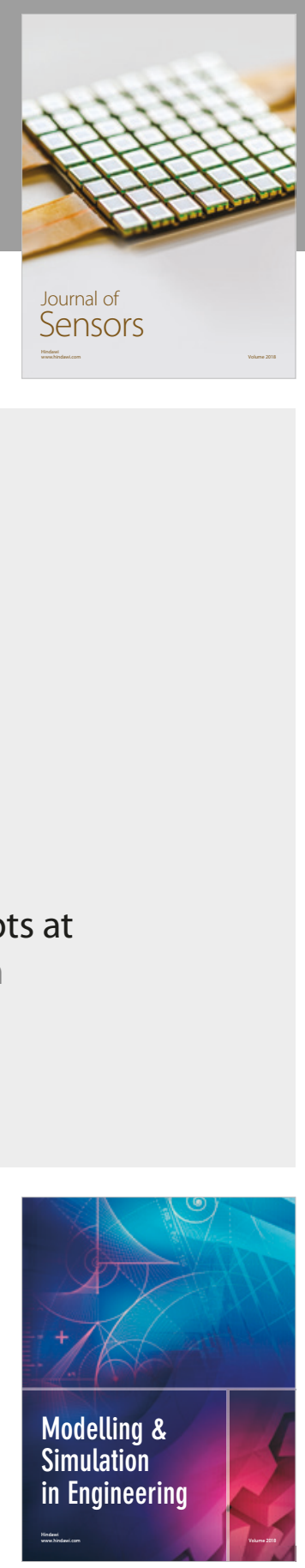

\section{Advances \\ Multimedia}
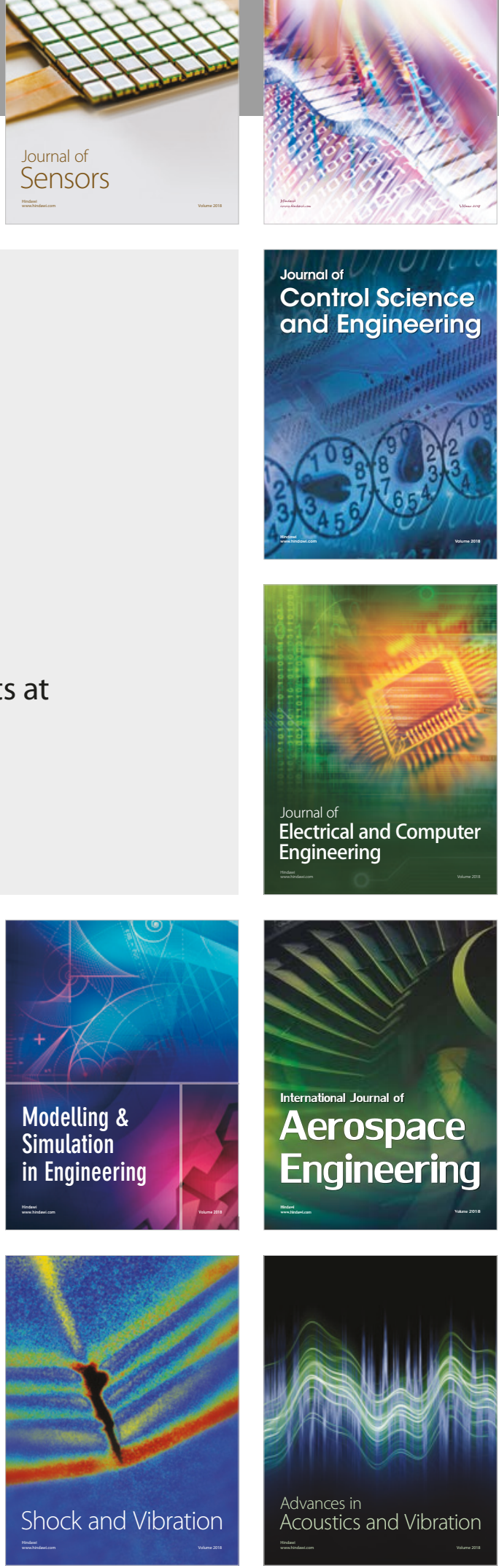